\title{
Hommage au Professeur Ogobara Doumbo
}

\author{
Colloque organisé par le COPED (Comité pour les pays en développement) de l'Académie des \\ sciences - 11 avril 2019, Fondation Simone et Cino del Duca*
}

\section{Tribute to Professor Ogobara Doumbo \\ Symposium Organized by the COPED (Committee for Developing Countries) of the French Academy of Sciences - April 11, 2019, Simone and Cino del Duca Foundation}

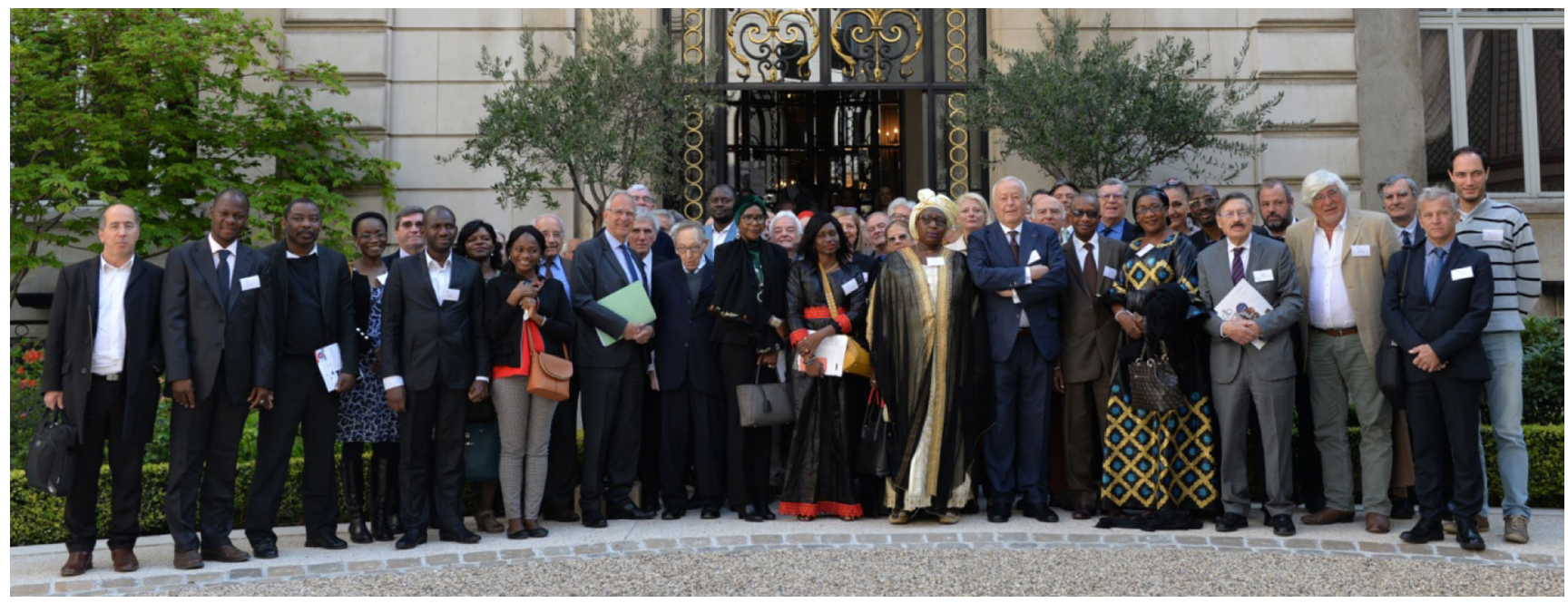

Fig. 1 Colloque du Coped de l'Académie des sciences, 11 avril 2019, Paris, France

\section{Introduction}

\section{B. Surugue, G. de Marsilly, J.-P. Chippaux}

Institut de recherche pour le développement ; MERIT, Institut Pasteur ; CRT, 28, rue du Docteur-Roux, Paris, France

bernard.surugue@gmail.com, gdemarsily@aol.com, jean-philippe.chippaux@ird.fr

Jeune médecin affecté au sud du Mali, Ogobara Doumbo participe très tôt aux travaux sur l'onchocercose, la schistosomose et la dracunculose ; à cet égard, on lui doit la certification de l'éradication de ce fléau dans de nombreux pays d'Afrique de l'Ouest. Mais le paludisme est la parasitose la plus grave en Afrique subsaharienne avec, selon l'OMS, plus de $90 \%$ des 400000 décès annuels recensés dans le monde. Pour vaincre chaque obstacle, Ogobara Doumbo complète d'abord sa formation, forgeant ainsi sa capacité à

\footnotetext{
* 16, rue Alfred-de-Vigny, F-75008 Paris, France
}

fédérer les compétences, convaincre les plus hautes instances et fonder une "École ». Selon cette approche, il crée avec l'entomologiste Yéya Touré le Centre de recherche et de formation sur le paludisme (MRTC) à Bamako, un dispositif d'excellence doté d'un réseau de villages sentinelles. Avec de solides arguments et une méthodologie associant les stratégies de luttes antivectorielles actives et passives, les traitements préventifs intermittents chez les femmes enceintes et les enfants préscolaires, la prise en charge des infections, le choix des molécules antipaludiques, les vaccinations, etc., le professeur Ogobara Doumbo, homme de défi, a fait de l'éradication du paludisme une question d'éthique et de solidarité internationales.

Nous présentons ici les résumés des communications qui ont célébré la personnalité et l'œuvre du professeur Ogobara Doumbo, soulignant les principales thématiques scientifiques de sa carrière. Le symposium a également permis de tracer les perspectives de l'héritage laissé aux jeunes générations engagées dans la lutte contre le paludisme et les maladies tropicales négligées. 


\section{Introduction}

Young medical doctor in southern Mali, Prof. Ogobara Doumbo took an early part in the studies on onchocerciasis, schistosomiasis, and dracunculiasis. In this respect, he is credited with certifying Guinea Worm eradication, a dreadful scourge in many West African countries. However, he was also associated in many studies on malaria, the most serious parasitic disease in sub-Saharan Africa where more than $90 \%$ of the 400,000 annual deaths worldwide occur, according to WHO.

To overcome each obstacle, Ogobara Doumbo first completed his training, strengthening his capacity to federate skills, convince the highest authorities and found a real school of research, practice and training. According to this approach, he created with the entomologist Prof. Yéya Touré of the Malaria Research and Training Center (MRTC) in Bamako, a device of excellence with a network of sentinel villages. With strong arguments and a methodology combining active and passive vector control strategies, intermittent preventive treatments for pregnant women and pre-school children, infection management, choice of antimalarial drugs, vaccinations, etc. Prof. Ogobara Doumbo, a man of challenge, has made the eradication of malaria an issue of ethics and international solidarity.

We present here the abstracts of the communications that celebrated the personality and the work of Prof. Ogobara Doumbo putting into perspective the main scientific themes of his career. The symposium also traced the perspectives of the legacy left to the younger generations engaged in the fight against malaria and neglected tropical diseases.

\section{Hommage à Ogobara Doumbo}

\section{A. Mérieux}

Président de la Fondation Mérieux

Alain.MERIEUX@institut-merieux.com

J'ai rencontré le Pr Ogobara Doumbo pour la première fois en 2003.

Mon fils, le Dr Christophe Mérieux, et Youssouf Issabré, l'un de nos collaborateurs biologistes maliens, s'étaient rendus au Mali pour analyser les besoins de ce pays en microbiologie clinique. De cette mission, ils sont rentrés convaincus de la nécessité de créer un laboratoire de diagnostic de référence. Entre-temps, Christophe avait rencontré Ogobara, et ils travaillaient ensemble à un projet de recherche commun. Pour que ce programme aboutisse, il fallait la bénédiction des Anciens, et c'est en cette qualité que j'ai « béni » ce projet à Lyon en 2003. C'était la première fois qu'Ogobara et moi nous nous rencontrions. Cela a été le début d'une magnifique collaboration au service de la santé publique du
Mali et plus largement des pays défavorisés. Et surtout le début d'une amitié exceptionnelle.

La Fondation Mérieux a inauguré à Bamako le laboratoire de diagnostic Rodolphe-Mérieux en 2005. Ogobara nous a ensuite demandé de créer une maternité. Nous sommes allés ensemble dans les faubourgs de Bamako, et nous avons présenté ce projet aux Anciens qui l'ont accepté. Je suis revenu inaugurer la maternité Michel-Galy en 2012 dans une période difficile pour ce pays.

À la mort de Christophe, nous avons créé, avec Pierre Messmer, le prix Christophe Mérieux décerné chaque année à l'Institut de France. Ogobara a été le premier récipiendaire de ce prix qui lui a été remis sous la Coupole en 2007 par le Pr François Gros. Ce jour-là, Pierre Messmer a demandé à Ogobara Doumbo de répondre, ce qui ne se fait jamais en de telles occasions. Ogobara a prononcé un discours extraordinairement émouvant.

Je n'ai jamais oublié les paroles de ce « fils de paysan dogon », c'est ainsi qu'il se présentait, qui après de brillantes études de médecine à Bamako, en France et aux États-Unis a choisi de revenir dans son pays pour lutter avec les siens contre le fléau du paludisme. Je n'ai pas oublié ses paroles sur Christophe qui était un frère pour lui. Ogobara est devenu un fils pour moi. Nous ne nous sommes jamais quittés depuis.

Ogobara Doumbo a joué un rôle déterminant dans l'évolution de la Fondation Mérieux. Membre de notre conseil scientifique et président du conseil d'administration du centre d'infectiologie Charles-Mérieux du Mali, il a également été le premier titulaire de la chaire « Docteurs Mérieux » à l'Institut hospitalo-universitaire Méditerranée infection à l'hôpital de la Timone à Marseille.

Il y a trois ans, en présence des Premiers ministres chinois et français, la Fondation Mérieux, le centre d'infectiologie Charles-Mérieux du Mali et l'Académie chinoise des sciences médicales signaient un accord de recherche pour lutter contre les maladies infectieuses au Mali. Ogobara, homme clé de la biologie clinique en Afrique, a joué un rôle majeur dans cet accord.

En 2017, alors que nous célébrions les 50 ans de la Fondation, Ogobara, en tant que témoin, nous apporta sa vision éclairante de la médecine avec un grand principe : faire non pas « pour ", mais « avec », les acteurs locaux, aussi bien dans la conception des projets de recherche que dans le renforcement des infrastructures de biologie et de soins. En favorisant l'accès à des soins de qualité pour tous et en développant la recherche locale en collaboration avec la communauté scientifique internationale, Ogobara Doumbo a fait avancer l'Afrique sur le chemin de l'autonomie.

Sa perte est immense, mais sa vision demeure, portée par les nouvelles générations de scientifiques africains.

Je rends hommage à la force de son humanisme, à sa grande simplicité et à l'incroyable charisme d'un homme 
dont la vision et l'action préfigurent l'avenir de la santé sur le continent africain et dans le monde. Je salue un ami.

\section{Hommage à Ogobara Doumbo}

\section{Gentilini}

Président honoraire de l'Académie nationale de médecine, 16, rue Bonaparte, F-75006 Paris, France

marc.gentilini@opals.asso.fr

Pourquoi la mort?

Un brillant journaliste français, Pierre Viansson-Ponté, avait autrefois répondu à cette question en affirmant « Toute mort est échec. »

Nous sommes nombreux, dans cette salle, à nous être déjà posé cette question, confrontés à la mort d'un être cher, plus jeune, brillant, engagé dans une vie active pleine de promesses. Nous ne culpabilisons pas pour autant pour notre survie et ne sombrons pas davantage dans le syndrome du survivant.

Pourquoi la mort ?

Ogobara Doumbo, le Pr Ogobara Doumbo, Ogo plus simplement, ne s'est peut-être pas posé cette question pour luimême, mais sûrement à propos des populations du Mali, plus particulièrement des enfants très jeunes mourant de paludisme à Plasmodium falciparum, paludisme saisonnier et mortifère dans cette région du Sahel où il a tant travaillé.

Sa vie durant, Ogobara s'est préoccupé de la mort des autres, de la mort des jeunes.

Fils de paysan dogon, profondément dogon, tradithérapeute, Ogobara est né un $1^{\mathrm{er}}$ janvier, mais d'année incertaine : 1955 ou 1956, approximation qui n'était pas pour lui déplaire.

Repéré par un instituteur pour son intelligence, il fut orienté vers l'enseignement supérieur, mais il avait, dès l'abord, tout pour comprendre à la fois le terrain d'où il venait, les thérapeutiques locales, empiriques ou non, et l'apport incontestable de la science; à condition toutefois qu'elle reste en lien avec le terrain et la société civile et que l'approche des maladies soit prise dans son ensemble, celui de tout l'être humain et celui de la société dans laquelle il vit, grandit, travaille, transmet et meurt.

Ogobara fit ses études à l'école de médecine de Bamako, à la faculté, dans la première décennie de son ouverture. Il y rencontra différents maîtres et fit preuve d'une capacité de travail et d'un engagement personnel exceptionnels. C'est son professeur de parasitologie, à Bamako d'abord puis à Marseille, Philippe Ranque, qui l'intégra plus tard dans son équipe méridionale où il obtiendra sa spécialité de parasitologie médicale. Il resta toujours très fidèle à Philippe Ranque et lui rendit un vibrant hommage en 2017.

Alliant la rigueur scientifique et l'humilité, un trait de sa personnalité, recherchant et développant le travail en équipe,
Ogobara sut construire, avec des praticiens formés à Bamako, un ensemble cohérent, efficace et passionné dans la lutte contre le paludisme afin de créer une recherche compétitive au sein d'un pays sahélien pauvre, considéré jusqu'à présent par des bureaucrates internationaux, « des experts », comme non éligible pour la recherche.

À tous ceux qui doutaient, à tous ceux qui niaient, il infligea un cinglant démenti, créant, après une formation approfondie en France et aux États-Unis, un centre de recherche sur le paludisme saisonnier et intermittent, axé en particulier dans la lutte contre cette affection chez les enfants de moins de cinq ans, tranche d'âge la plus vulnérable au paludisme, tragiquement mortel dans le Sahel.

Fort de l'appui coalisé de ses alliés et amis, il créa à Bamako un centre de recherche hautement performant : le Malaria Research and Training Center ou MRTC (centre de recherche et de formation sur le paludisme).

Le paludisme, la malaria qui tuait autrefois près d'un million d'individus par an, connaissait, depuis quelques décennies, une régression spectaculaire, et la mortalité restait cependant autour de 500000 personnes, enfants le plus souvent, décédées chaque année. "Pour moi, le paludisme a toujours été un drame. Cette maladie tue surtout les enfants, un jeune toutes les deux minutes. »C'est ce qu'il affirmait, avec raison, au début des travaux de son équipe. Plus tard, il ajouta " Au Mali, aucun enfant ne meurt du paludisme dans les villages où nous intervenons. »

Les villages, ses villages : "Si l'on veut combattre le paludisme, il faut revenir dans les villages... Ce qui fait notre force, c'est notre présence sur place avec les communautés de villageois et notre souci de répondre aux besoins quotidiens de la population. »

Courageusement, en dépit de l'instabilité politique et des drames de la guerre civile, sur une dizaine de sites répartis sur tout le territoire, il n'abandonna jamais, même ceux qui étaient particulièrement menacés par les terroristes. Optimiste, il installa ces deux éléments fondamentaux pour une prise en compte des problèmes de santé et leur maîtrise que sont l'apport de l'eau et celui de l'électricité. Parallèlement, il créa écoles, routes et même connexions internet au sein d'une population qui renforça, convaincue, les liens indispensables pour une action durable.

Ogobara Doumbo n'était pas qu'un chercheur, ô combien compétent et efficace en paludologie, il était avant tout un homme de santé publique, s'attaquant en priorité à une affection décimant la population infantile, mais qui faisait partie aussi d'un ensemble de maladies transmissibles dans un climat de précarité et de pauvreté. Lors d'une rencontre à Casablanca, il y a quelques années, à la table ronde que j'animais avec lui, il fit la démonstration de ses qualités plurielles qui rejoignaient, dans son engagement actuel, la formation initiale qu'il avait reçue sur le terrain au sortir de l'école de Bamako, en zone rurale, la « brousse » qu'il aimait tant. 
C'est cette dimension que nous devons souligner, et c'est la direction qu'il prenait résolument depuis quelque temps.

Un autre aspect de la personnalité de ce chef d'équipe, de ce grand patron, que je voudrais souligner, c'est l'esprit d'équipe qui l'anima jusqu'au bout et qui aujourd'hui permet d'espérer de ses successeurs la pérennisation de l'œuvre entreprise ; son développement même, témoignage de l'ancrage scientifique du travail accompli par son promoteur.

De toute façon, un centre comme le MRTC, sis à Bamako, a l'avantage sur tous les centres d'Europe ou d'Amérique d'avoir le contact direct avec la population et son impaludation.

L'équipe a reçu toutes les autorisations des structures internationales, scientifiques et éthiques pour effectuer sur le terrain des essais dans la perspective d'un vaccin contre le paludisme.

Celui-ci existera sans doute, un jour, efficace, accessible, inoffensif. Quand ? Bientôt? Plus tard ? Personne au juste ne peut en préciser la date, mais espérons une protection pour tous : d'abord les populations en zone endémoépidémique, ensuite les voyageurs.

Cet espoir, Ogobara l'avait, et les travaux de son équipe progressaient dans ce domaine. Prudemment, et avec humilité, il disait que «l'espoir est permis ». À vous, ses successeurs, de poursuivre dans cette voie et de prouver qu'il avait raison en dépit de la guerre d'usure qu'est le paludisme.

À Paris, le 29 mars 2016 avait été programmée à l'Académie de médecine, rue Bonaparte, une séance dédiée intitulée « L'élimination du paludisme dans le monde est-elle possible ? Médecins et chercheurs dans cette lutte », organisée par Martin Danis, j'avais été chargé de l'introduire.

Le premier intervenant prévu était Ogobara Doumbo pour ses travaux sur le paludisme saisonnier intermittent et la lourde mortalité qu'il entraînait chez les enfants de moins de cinq ans. Mais quelques heures avant son envol vers nous, un accident de la voie publique sur une route du Mali avait décimé une de ses jeunes équipes travaillant sur le terrain et entrainnant morts et blessés, nous privant ainsi de la présence d'Ogobara, retenu auprès des familles endeuillées. Cet épisode tragique nous avait beaucoup peinés à l'époque, car nous savons le prix d'un cerveau, le prix d'une vie et les conséquences pour les familles. Lors de cette séance, j'ai présenté les condoléances de l'Académie et assuré de notre sollicitude et de notre solidarité tout le groupe des chercheurs.

L'activité scientifique en paludologie du Pr Ogobara Doumbo et sa démarche en santé publique, " pour les Maliens et par les Maliens », ont été, à de nombreuses reprises, reconnues et récompensées.

Ce fut le cas en 2007, sous la coupole de l'Institut, par vous-même, Monsieur le Secrétaire perpétuel honoraire François Gros, en lui remettant le prix Christophe Mérieux de l'Institut de France.
Il a été récompensé en Espagne, par le prix du Prince des Asturies en 2008; par le prix d'excellence en santé publique du prince Mahidol de Thaïlande, en 2011 ; par le prix international de la recherche de l'Inserm, en 2013, décerné, pour la première fois, à un chercheur africain.

Ogobara était, par ailleurs, officier de la Légion d'honneur de France, la plus haute distinction de notre pays, et titulaire des Palmes académiques.

Chevalier de l'Ordre du Mali, il a été promu, lors des obsèques officielles nationales à Bamako, par le président de la République Ibrahim Boubacar Keïta, grand officier de l'Ordre national.

Beaucoup de membres présents dans cette salle, lors de son décès, ont dit leur tristesse et porté un jugement émouvant sur son parcours professionnel, les résultats obtenus et toujours sa modestie.

Vous l'avez fait, Jean-Paul Moati, président-directeur général de l'Institut de recherche pour le développement (l'IRD), en lui rendant un vibrant hommage.

Vous l'avez fait également, cher Président Alain Mérieux, en évoquant la force de son humanisme, sa grande simplicité et l'incroyable charisme d'un homme dont la vision et l'action préfigurent l'avenir de la santé sur le continent africain. Vous avez dit, au nom de votre Fondation, qu'il était, pour vous, un « conseiller exceptionnel ».

Il a su capitaliser, sur les forces de son pays, son expérience acquise au Mali puis en France au côté des familles de l'arrière-pays marseillais en fondant, en 1984, avec d'autres « Santé Sud », association de médecins de campagne qui recensent plus de 250 médecins maliens installés sur l'Hexagone et qui subviennent aux besoins de nos compatriotes.

Au plan international, il était fier d'avoir vu le modèle proposé pour la santé au Mali, adopté à Madagascar, au Bénin et en Guinée.

Le Pr Ogobara Doumbo a travaillé, toujours en lien avec son université du Mali, avec l'IRD, le centre d'infectiologie de Bamako et la Fondation Mérieux, mais aussi avec la Tulane School of Public Health à La Nouvelle-Orléans, aux États-Unis et à l'Institut hospitalo-universitaire Méditerranée de Marseille.

Il a travaillé avec l'Académie des sciences du Mali, l'Académie africaine des sciences et l'Académie de médecine de France où il a été élu membre correspondant étranger, en 2008, l'année de ma présidence. Toutes ces manifestations en sa faveur étaient avant tout, pour lui, la reconnaissance des activités de la cohorte de chercheurs qu'il avait réunis et dont je salue ici tous les membres présents dans cette salle, en la personne, entre autres, de son directeur actuel.

Mais en 2018, c'est le drame. Après une intervention chirurgicale efficace et bien conduite, par des mains expérimentées, maliennes, comme le souhaitait le Pr Ogobara Doumbo, des complications liées aux maladies nosocomiales survinrent. Sa santé brusquement s'aggrava. Il fut transporté 
d'urgence en réanimation à l'hôpital de la Timone à Marseille, sa seconde ville, où malheureusement il décéda le 9 juin 2018.

Avec son décès, les chercheurs africains sont un peu orphelins.

Au cours de cette journée du 11 avril 2019, presque un an plus tard, nous sommes réunis dans une communion avec sa personne, sa famille, son équipe, et solennellement les académies des sciences et de médecine de France rendent un hommage ému à cette disparition prématurée.

Dans la vie d'un hospitalo-universitaire et d'un chercheur, la vraie réussite est celle des autres, celle de ceux qui permettent de se relever, d'être relayé.

À quelques jours près, avant de prononcer ce rappel du parcours du Pr Ogobara Doumbo, grande et belle figure de la recherche d'Afrique subsaharienne, j'ai eu le privilège de rencontrer à nouveau le Dr Denis Mukwege, prix Nobel de la paix 2018, chirurgien-gynécologue qui « répare les femmes » atrocement meurtries par la soldatesque locale ou de pays voisins, qui les pourchasse et les viole autour du lac Kivu, en République démocratique du Congo. C'était au cours d'un colloque intitulé « Viols, armes de guerre ». J'aurai prochainement à représenter à nouveau le parcours courageux, efficace, sans cesse menacé de cette belle figure de la nouvelle Afrique.

Et je ne peux, ce matin, m'empêcher de rapprocher l'histoire de ces deux fils d'Afrique, celui du Sahel et celui de l'Afrique centrale, qui, tous deux, prouvent les extraordinaires ressources humaines du continent africain dans le domaine, en particulier, de la santé et de l'éthique, deux modèles pour la jeune Afrique, que nous accueillerons bientôt, je l'espère, elle aussi, à l'Académie de médecine de France.

Mesdames et Messieurs,

En ces heures douloureuses pour le Mali qui connaît des épisodes meurtriers entre ses fils, au moment où, tragiquement aussi pour nous, de France, qui défendons depuis toujours les pays amis d'Afrique, des manifestations de rue à Bamako qui nous blessent par leur injustice, leurs mensonges et leur violence, en ces temps douloureux dis-je, la voix d'hommes de paix, comme celle d'Ogobara Doumbo, eut été particulièrement utile pour rétablir la vérité, apaiser les angoisses et ramener chacun à la raison, en vue de construire une paix durable et une solidarité fraternelle, universelle.

«Pourquoi la mort ? », ai-je posé comme question en commençant ce rappel de la vie et de l'œuvre d'Ogobara Doumbo. À vous les héritiers de démontrer, par votre engagement, que le sien sera pérenne.

À vous de « faire votre part ».

À vous de faire aux autres ce qu'il a fait pour vous.

Toutes et tous ici, ce matin, nous sommes venus célébrer, avec foi, l'ardeur qui l'animait. Tous et toutes, nous le remercions, avec émotion et affection, pour ce qu'il a entrepris au service de son pays et à celui de tous les hommes de cette terre fragile.

Ogobara Doumbo est entré dans l'Histoire.

\section{Témoignage de Doumbo Safiatou Niaré, veuve d'Ogobara Doumbo}

\section{S. Niaré}

Professeure agrégée en parasitologie-mycologie, université des sciences, des techniques et de technologie de Bamako, Mali sdoumbo@icermali.org

C'est pour moi un réel plaisir et une grande émotion de prendre la parole devant cette auguste assemblée pour témoigner à l'endroit d'un monsieur, un monsieur pas comme les autres pour la famille Doumbo. Il s'agit de feu Ogobara K. Doumbo.

Je voudrais tout d'abord remercier chaleureusement les initiateurs et les organisateurs de ce colloque de nous avoir réunis ce matin.

Nous remercions tous les amis, collègues, étudiants, parents, d'avoir fait le déplacement.

Nos chaleureux remerciements vont à ceux qui, de loin ou de près, suivent cette séance avec nous ce matin.

De la phase préparatoire à la finalisation de l'agenda du colloque, de l'organisation de notre voyage à l'accueil chaleureux qui nous a été réservé, nous avons pu mesurer combien vous aviez été attachés à Ogo.

Nous avons été affectueusement marqués par les témoignages de sympathie de toutes les nations, notamment celle du Mali et particulièrement par le témoignage du président de la République, Son Excellence Ibrahim Boubacar Keïta ; depuis le décès d'Ogo jusqu'à ce matin, notre famille remercie sincèrement toutes les personnes qui, par leur présence, leur soutien et leur affection se sont associées à notre peine.

Cher Ogo, en ce jour de témoignage rendu, je te sais déjà réjouis de l'événement, c'est-à-dire le colloque scientifique tenu ici en France que tu as voulu toujours animer avec amour dans le partage du savoir et dans l'annonce des perspectives avec des visions de dix ans en avance. Oui, la vie d'Ogo se résumait en cela, « donner et recevoir », la mise à jour des connaissances scientifiques et l'anticipation des solutions aux problèmes. Le pays d'organisation de ce colloque ne pouvait pas être mieux choisi, car Ogo a toujours été animé d'admiration pour ses patrons, collègues, amis et parents français. La preuve est que c'est dans ce beau pays où il a étudié, collaboré, travaillé et enfin vécu les derniers jours de sa vie, qu'il rendit l'âme, précisément à Marseille, sa ville d'amour.

Le décès d'Ogo a été à la fois de la stupeur parce que rien ne laissait présager cette disparition très brutale et très prématurée, puis c'est aussi beaucoup de tristesse parce que c'était une personnalité exceptionnelle.

Nous sommes tous là aujourd'hui, la famille, les amis et ceux qui l'ont suffisamment approché pour apprécier l'étendue de sa personnalité et de sa bonté. 
Ogo était « un homme particulièrement attachant », « à l'intelligence rare », « au tempérament chaleureux et généreux », « entier et absolument passionné ». « Passionné surtout par la science, son pays et sa grande famille, Ogo a consacré toute sa vie, toute l'étendue de ses talents et la force exceptionnelle de ses convictions à la science ». Oui, il était surtout un homme modeste et humble.

Il était une personnalité forte, complexe, capable de résister en toutes circonstances ; pour lui, le négatif n'avait pas sa place dans la vie d'un homme, tout devait être possible en relativisant seulement selon le contexte. Tout au long de sa carrière, grâce à votre collaboration et votre accompagnement, il a obtenu des prix (au Mali, en Afrique, en Europe, en Amérique, etc.) qu'il a su partager avec son équipe, mais surtout avec sa famille, car d'après lui, tout se gagne en équipe.

La vie d'Ogobara Doumbo a été marquée par un idéal qui correspondait au développement de la médecine, de la formation des jeunes médecins et chercheurs, de la recherche scientifique et surtout du partage de connaissances en famille, au Mali, en Afrique et dans le monde entier sans considération aucune. Il a été soutenu par vous tous ici présent (en le formant, en lui octroyant des possibilités de formation pour lui, son équipe, mais surtout sa famille). Il a été une inspiration pour ses étudiants, un pionnier pour ses pairs et une fierté pour la nation malienne.

En plus de ses travaux de recherche, Ogo savait créer du temps pour le social de par sa présence aux événements sociaux en famille, des voisins du quartier, des amis et collègues, etc. Ses collaborateurs, ses amis et connaissances pourront confirmer avec moi qu'Ogo a toujours été présent quand il le fallait, parfois par surprise.

Durant tout le temps que nous avons vécu avec lui en famille, nous avions ensemble fait tant de choses. Nous avons mangé, bu avec lui, nous avons voyagé, partagé les soucis et les travaux quotidiens. Avec lui, nous avons partagé tant de projets et tant d'espoirs. Et voilà que maintenant il nous quitte. Il y a tant de choses encore que nous aurions voulu faire ensemble.

Ogo a toujours géré la famille biologique de près ou de loin avec amour, attention, tendresse et délicatesse dans le détail (il n'avait que des reines, des princes et des princesses en famille). Il fut un ami, un confident, un collègue et un patron, pour moi particulièrement. Il a été ce père de famille irréprochable et un mari hors pair. Il fut celui qui n'était content que quand il partageait avec les autres, même en séjour d'hôpital : pendant le mois béni de carême, il ordonna comme à l'accoutumée d'offrir des vivres aux nécessiteux ainsi qu'à qui de droit selon la tradition. Toute la famille Doumbo s'en souviendra et exécutera selon sa volonté. Ogo n'a jamais su élever la voix en famille et a toujours discuté avec ses enfants d'homme à homme. En tant qu'épidémiologiste, Ogo aimait répéter à ses enfants «dites-vous bien mes chers enfants : ce n'est pas la quantité de temps que l'on peut passer ensemble, mais surtout la qualité », et ses enfants savaient apprécier les bons moments passés à ses côtés. Oui, c'était aussi de ses compétences en biostatistique, en probabilité et en épidémiologie qu'il a toujours enseignées à ses étudiants, ses collaborateurs et dans les normes de l'éthique et de la déontologie. Quand il avait une opinion, il acceptait d'en débattre avec les autres. Il plaçait le débat à un haut niveau à la fois de conviction et de talent. C'était une personnalité qui a enrichi notre vie de famille.

Mais tout cela semble se ralentir le 6 mai 2018 et s'arrêter ce matin du 9 juin 2018 où j'étais présente auprès de lui, impuissante devant la volonté divine, récitant les versets du Coran comme il se doit sur un mourant selon la religion musulmane.

Nous, la famille, voudrions nous souvenir de lui, continuer de travailler à tout ce qu'il attendait, à tout ce qu'il espérait, ne jamais faillir à notre devoir.

Comme un mur, la mort nous sépare de lui comme le souffle du vent qui balaie les obstacles.

Notre amitié, notre affection et notre espérance s'en iront le rejoindre là où désormais il nous attend près de Dieu.

En effet, l'accompagnement d'Ogo tout au long de sa brève, mais douloureuse maladie a été une épreuve pour nous, tant nous avions de la peine de le voir souffrir et s'en aller petit à petit, particulièrement la dernière semaine de sa maladie.

Notre consolation est de savoir qu'il a été très bien soigné par l'équipe médicale qui avait la responsabilité de son traitement, mais également que vous avez été nombreux, famille, amis, voisins, collaborateurs à lui rendre visite ou à lui envoyer un signe d'amitié, ce qui lui a été très réconfortant.

N'avez-vous pas écrit que « Le grand baobab est tombé. Trop tôt, trop brutalement. L'enseignement supérieur, la recherche et la science africaine sont ébranlés », j'ajouterai au nom de la famille que le chameau a déposé le poids ; je le traduis en Bamanan « Nionkome Ye Doni Djigui. »

Oui, il est parti de sa belle mort. Dors en paix, Ogo, dans le jardin du paradis, Firdaws. Nous te regretterons à jamais.

\section{Les maladies infectieuses au début du $\mathrm{XXI}^{\mathrm{e}}$ siècle}

\section{Kindé-Gazard}

Ancienne ministre de la Santé publique, présidente de la Société ouest-africaine de parasitologie, université d'Abomey-Calavi, faculté des sciences de la santé, Cotonou, Bénin

darskg2012@gmail.com 


\section{Introduction}

Les maladies infectieuses (MI) sont provoquées par la transmission d'un micro-organisme ou d'un agent infectieux (virus, bactérie, parasite, champignon, prions). La plupart des pathogènes évoluent avec leur hôte depuis des millénaires, ce qui confère une stabilité relative de leur équilibre dynamique au sein des écosystèmes.

Ces maladies sont plus ou moins contagieuses (la transmission d'homme à homme de la tuberculose, la toxiinfection du tétanos, l'implication d'un hôte intermédiaire dans la transmission du paludisme, les affections sexuellement transmissibles comme le sida). Les facteurs favorisants relèvent de l'épidémiologie, de l'écoépidémiologie, de la localisation (ports et aéroports, notamment). Les profonds changements que nous faisons subir à notre environnement ont des conséquences importantes sur la propagation des MI. Les changements climatiques, les nouvelles chaînes alimentaires et industrielles, les déplacements massifs des populations grâce au développement des moyens de transport rapides, la destruction et la dégradation des écosystèmes transformant le milieu de cohabitation des populations animales et humaines sont à l'origine de nouvelles maladies émergentes, réémergentes et des épidémies.

\section{Origine des maladies infectieuses}

Jobling et al. [3], Wolfe et al. [8] et Morand et al. [4] affirment qu'il n'y a pas de réponse absolue quant à l'origine des MI. Cependant, quelques données historiques sont édifiantes, comme l'origine de la syphilis en Europe ou la peste de Thèbes durant l'Antiquité pour ne citer que celles-là. Quatre maladies vont permettre d'illustrer quelques-uns des schémas d'origine possible des MI : la rougeole, la rage, la peste (maladies anciennes), le SRAS (syndrome respiratoire aigu sévère, maladie émergente). Si la rougeole, la rage et le SRAS sont d'origine virale, la peste est quant à elle d'origine bactérienne.

- La rougeole est causée par un virus de la famille des Paramyxoviridae et du genre Morbillivirus. Il serait issu de l'adaptation du virus de la peste bovine lors du processus de domestication des Bovidés entre les $\mathrm{XI}^{\mathrm{e}}$ et $\mathrm{XII}^{\mathrm{e}}$ siècles [2];

- la rage est causée par un virus de la famille des Rhabdoviridae du genre Lyssavirus : longtemps associée au chien, on admet désormais qu'il s'agit d'un rhabdovirus d'insecte qui se serait adapté aux chiroptères (chauvessouris), qui serait le réservoir originel [6] ;

- le SRAS est causé par un virus de la famille des Coronavirus, le Sars-CoV. Le réservoir animal du coronavirus du SRAS a été identifié comme étant une chauve-souris insectivore. L'hôte intermédiaire qui a permis le passage du virus à l'Homme est la civette palmiste (Paguma larvata), animal sauvage vendu sur les marchés et consommé au sud de la Chine [7];

- la peste : tout le monde se souvient de l'adage qui dit « qui dort avec son chien se lève avec des puces ». Ce ne sont cependant pas les puces du chien qui sont responsables de la transmission de Yersinia pestis, mais celles du rat (Xenopsylla cheopis puce de Rattus rattus et Rattus norvegicus), autres commensaux de l'homme... Une théorie récente incrimine les ectoparasites humains, notamment les poux (Pediculus humanus), pour expliquer la diffusion explosive de la peste lors de la deuxième pandémie [1].

Ces données montrent la complexité de l'origine de l'agent infectieux, de sa rémanence dans l'environnement, de son passage à l'homme - et entre les hommes euxmêmes - et de sa virulence. Face aux risques sanitaires à l'échelle mondiale, la gouvernance de la santé a évolué vers une approche one health, fondée sur la théorie one medicine [5], exposée pour la première fois en 1984 par Calvin Schwabe dans son ouvrage Veterinary Medicine and Human Health où il préconise une réponse aux zoonoses combinant médecine humaine et médecine vétérinaire. En effet, $75 \%$ des maladies nouvellement apparues sont provoquées par des agents infectieux d'origine animale : sida (chimpanzé et mangabey), encéphalopathie spongiforme bovine (bovins), grippes (oiseaux, animaux aquatiques), SRAS (chauves-souris), trypanosomoses à Trypanosoma cruzi (plusieurs espèces de mammifères), à Trypanosoma brucei (ruminants sauvages et domestiques), paludisme à Plasmodium knowlesi (parasite du singe macaque).

\section{Problématique des maladies infectieuses au $\mathrm{XXI}^{\mathrm{e}}$ siècle}

On observe, malgré le recul majeur des grandes MI (variole, syphilis, diphtérie), le développement d'un grand nombre de pathogènes à caractère zoonotique et la mondialisation du risque malgré les progrès de la médecine, le développement exponentiel de la résistance de nombreux micro-organismes aux antimicrobiens ou de vecteurs aux insecticides.

Ainsi, en octobre 2008, l'Organisation mondiale de la santé (OMS), l'Organisation des Nations unies pour l'alimentation et l'agriculture (FAO) et l'Organisation mondiale de la santé animale (OIE) ont présenté dans un document influent, intitulé "Contribuer à One World One Health », un plan stratégique pour réduire les risques de MI à l'interface des écosystèmes humains et animaux.

\section{Évolution des maladies infectieuses}

Charles Nicolle, en 1930, dans son livre Naissance, vie et mort des maladies infectieuses, indique qu'" Il naîtra de nouvelles maladies; il en disparaîtra lentement quelques- 
unes, sans cesse des problèmes nouveaux se poseront. Les errements de l'humanité, l'hygiène et la prévention sont les facteurs essentiels qui gouvernent le destin des maladies infectieuses. »

Depuis lors, il faut signaler :

- la découverte de 335 nouvelles MI entre 1940 et 2004 ;

- sur 1407 agents microbiens et parasitaires pathogènes pour l'Homme, $61 \%$ étaient d'origine animale ;

- le nombre de virus, dont certains particulièrement virulents, découverts depuis la seconde moitié du $\mathrm{XX}^{\mathrm{e}}$ siècle (chikungunya, Ebola, Lassa, Marburg, Monkeypox, VIH, Zika...).

Parmi elles, une majorité de maladies bactériennes (brucellose, fièvre charbonneuse, botulisme, fièvre $\mathrm{Q}$, leptospirose, listériose, maladie des griffes du chat, maladie de Lyme, pasteurellose, peste, pseudotuberculose, salmonellose, tétanos, tuberculose, etc.) et virales (fièvre de la vallée du Rift, rage, grippes, fièvre de Lassa, maladie à virus Ebola, dengue, fièvre jaune, chikungunya, maladie de Newcastle, Pox virus, virus Crimée-Congo, etc.) sont zoonotiques.

\section{Défis}

Le principal défi passe par la promotion du dialogue entre différents acteurs (santé, recherche, sociologie, économie, pouvoirs publics, citoyens) ainsi que le partenariat entre les secteurs public et privé.

Il comprend aussi la promotion de la recherche sur les maladies (infectieuses ou pas) émergentes (médicale, épidémiologique, socio-économique et écologique). Les domaines de recherche s'étendent de l'étude des MI zoonotiques aux problèmes prioritaires tels que :

- la mise au point de nouvelles molécules face à la résistance des bactéries aux antibiotiques, des parasites aux antiparasitaires ;

- la toxicologie + écotoxicologie (santé environnementale) ;

- les pathologies associées à des expositions à des substances chimiques ;

- la problématique des infections nosocomiales ;

- la menace bioterroriste.

Un important défi en termes de perspectives, est de proposer un système de surveillance, d'anticipation (aux niveaux régional, national et international) et de réponse aux émergences.

\section{Sécurité sanitaire internationale - partenariat/ coopération internationale}

Préparé depuis 1969, le Règlement sanitaire international (RSI) a été adopté en 2005 et régulièrement amendé par la suite. Il définit la conduite approuvée pour prévenir et com- battre les menaces, il permet de mettre en place des outils d'alerte et d'organiser la gouvernance des systèmes de santé.

Il prévoit de développer des outils de communication et d'information des populations du Nord comme du Sud sur les pathologies infectieuses en s'aidant des nouveaux médias et en formant les ressources humaines en santé, notamment dans les pays du Sud.

\section{Conclusion}

La santé est un concept global qui prévoit de vaincre les maladies, de faire face aux menaces sanitaires, de soigner le plus grand nombre et de s'attaquer aux inégalités (Bernard Jégou). Face aux MI émergentes au XXI ${ }^{\mathrm{e}}$ siècle, il faut s'appuyer sur les événements historiques marquants : la peste et la quarantaine, le choléra et l'assainissement, la variole et la vaccination...

Il importe de définir les priorités de recherche sur les MI (médicaments, vaccins) et d'encourager la coopération internationale.

\section{Références}

1. Dean KR, Krauer F, Walløe L, et al (2018) Human ectoparasites and the spread of plague in Europe during the Second Pandemic. Proc Natl Acad Sci USA 115:1304-9. doi: 10.1073/pnas.1715640115. Epub 2018 Jan 16

2. Furuse Y, Suzuki A, Oshitani H (2010) Origin of measles virus: divergence from rinderpest virus between the 11th and 12th centuries. Virol J 7:52. doi: 10.1186/1743-422X$7-52$

3. Jobling MA, Hurles ME, Tyler-Smith C (2003) Human evolutionary genetics: origins, peoples and disease. Garland Science, New York, 458 pp. ISBN 9-780815-341857

4. Morand S, Jittapalapong S, Suputtamongkol Y, et al (2014) Infectious diseases and their outbreaks in AsiaPacific: biodiversity and its regulation loss matter. PLoS One 9:e90032. doi : 10.1371/journal.pone.0090032. eCollection 2014.

5. Papadopoulos A, Wilmer S (2011) Introduction au concept «Une seule santé ». Centre de collaboration nationale en santé environnementale, $10 \mathrm{p}$

6. Troupin C, Dacheux L, Tanguy M, et al (2016) Largescale phylogenomic analysis reveals the complex evolutionary history of rabies virus in multiple carnivore hosts. PLoS Pathog 12:e1006041. doi:

10.1371/journal.ppat.1006041. eCollection 2016 Dec

7. Wang LF, Eaton BT (2007) Bats, civets and the emergence of SARS. Curr Top Microbiol Immunol 315:325-44

8. Wolfe ND, Dunavan CP, Diamond J (2007) Origins of major human infectious diseases. Nature 447:279-83 


\section{Rôle de la recherche, de la formation et du partenariat pour faire face aux défis de l'élimination des maladies parasitaires en Afrique}

\section{O. Gaye}

Professeur de parasitologie, faculté de médecine, université Cheikh-Anta-Diop de Dakar

oumar.gaye@ucad.edu.sn

Présentes en Afrique depuis de très nombreuses années, les maladies infectieuses et parasitaires comme la variole, le paludisme, la fièvre jaune, les trypanosomoses ont été prises en charge en Afrique de l'Ouest et du Centre par les pionniers Le Dantec, Jamot, Muraz, Richet... L'École africaine de médecine Jules-Carde, créée en 1918 à Dakar, a posé les jalons de la politique de formation et de recherche dans cette région de l'Afrique. En 1958, l'École de médecine est érigée en faculté ; les cadres supérieurs et intermédiaires sont formés pour prendre en charge les préoccupations sanitaires des populations africaines. L'apport des Prs Gentilini, Larivière, Carme, Ripert, Juminer, Mouchet a été déterminant dans la mise en œuvre de la nouvelle politique de formation et de recherche médicales en Afrique de l'Ouest et du Centre, politique que vont continuer et renforcer d'éminents enseignants-chercheurs parmi lesquels les Prs Diallo, Sadeler et Assale. Les organismes de coopération sanitaire régionale comme l'OCCGE et l'OCEAC et leurs institutions de recherche et de formation (le centre Muraz de Bobo Dioulasso, l'institut Pierre-Richet de Bouaké, le CERMES de Niamey), l'Orstom, les instituts Pasteur ainsi que les universités vont jouer un grand rôle dans la politique sanitaire de contrôle des grandes endémies. Des plans nationaux de lutte sont élaborés avec comme objectif final l'élimination du paludisme, des bilharzioses, de l'onchocercose, de la filariose lymphatique, de la trypanosomose humaine africaine (THA), de la dracunculose.

Ainsi, la THA a été endiguée de 1960 à 1965 par des équipes mobiles; elle a ressurgi dans les années 1980. Un nouveau programme avec une surveillance plus étroite a permis de dépister 300000 cas en 1995 et 5000 en 2010.

Le programme d'élimination de la filariose lymphatique pour 2020 cible 30 pays en Afrique.

Concernant l'onchocercose, le Programme de lutte contre l'onchocercose en Afrique de l'Ouest (OCP) a lancé, dès 1975 , les opérations de lutte antivectorielle de grande envergure avec les pulvérisations des gîtes larvaires et initié, au début des années 1990, le traitement à grande échelle des populations à risque par l'ivermectine. Par la suite, le Programme africain de lutte contre l'onchocercose (APOC) a organisé et généralisé la distribution d'ivemectine à base communautaire. Une couverture géographique à $100 \%$ et une couverture thérapeutique à $75 \%$ avec le praziquantel sont requises pour la chimioprévention des bilharzioses ; à côté du traitement, l'assainissement, la disponibilité d'une eau potable et la lutte contre les mollusques seront prioritaires pour l'élimination des bilharzioses.

Pour toutes ces parasitoses, les résultats obtenus ont été le fruit d'une collaboration entre les scientifiques, les programmes nationaux, les communautés, les partenaires au développement, notamment avec la fourniture gratuite des médicaments lors des campagnes de chimiothérapie préventive de masse. Les taux de couverture géographique et thérapeutique ont progressé ; des résultats majeurs sont notés comme au Togo, le premier pays d'Afrique subsaharienne à avoir éliminé la filariose lymphatique, au Maroc et en Tunisie où les schistosomoses ont été éliminées ; la dracunculose est sur le point d'être éradiquée, car il ne reste que 28 cas notifiés en 2017 au Tchad, en Éthiopie et au Soudan du Sud.

Plusieurs initiatives prises au niveau mondial et en région africaine ont réaffirmé le caractère prioritaire de l'élimination des maladies tropicales négligées (MTN), dont une bonne partie est constituée de parasitoses humaines. Citons l'objectif 3 du développement durable « Mettre fin aux MTN d'ici 2030 », la Déclaration de Londres en janvier 2012 «Éliminer dix MTN en 2020 », le lancement en 2016 du projet " Expanded Special Project for Elimination of NTDs » (EPEN) par l'OMS Afrique ou l'engagement du G7 en 2015.

Les défis à l'élimination de ces endémies parasitaires restent cependant importants. Les modifications du climat, la forte poussée démographique, la mauvaise urbanisation et les déplacements de populations constituent des facteurs favorisant l'introduction de nouveaux pathogènes, l'émergence de nouvelles zoonoses, l'apparition d'espèces hybrides et la résistance des parasites. D'autres types de défis aussi importants, tels que l'insuffisance en ressources humaines de qualité, la faiblesse des systèmes de surveillance, le manque de coordination entre les institutions et l'insuffisance ou le manque de financement, menacent l'objectif d'élimination des endémies parasitaires. Pour y faire face, la collaboration entre les ministères de la Santé, les scientifiques, les partenaires et le secteur privé sera une priorité. Le renforcement des capacités avec une adaptation des programmes de formation aux défis sanitaires actuels sera au centre. C'est ainsi que les universités ont mis en place des diplômes d'études spécialisées (DES), de master, de $\mathrm{PhD}$ et de post-doc. Héritiers de leurs maîtres, les Prs Same Ekobo au Cameroun, Kombila au Gabon, Guiguemde au Burkina Faso, Koné en Côte-d'Ivoire, Massougbodji au Bénin, Doumbo au Mali, ainsi que Ndir, Faye Ousmane et Gaye au Sénégal, avec leurs différentes équipes et partenaires scientifiques, se sont impliqués pour relever les défis, d'une part, par l'orientation de leurs enseignements et travaux de recherche et, d'autre part, en accompagnant les programmes nationaux dans la formulation et la mise en cuvre de leurs stratégies de lutte. 
Afin de renforcer la collaboration scientifique, plusieurs réseaux de recherche et de formation sont constitués : le West African Global Health Alliance (WAGHA), le West African Network for Tuberculosis, Aids and Malaria (WANETAM), le Central African Network for Tuberculosis Aids and Malaria (CANTAM), l'African Network on Vector Resistance (ANVR) et, plus récemment, le programme Developping Excellence in Leadership, Training and Science in Africa (DELTAS Africa); ce dernier programme, qui regroupe 11 institutions africaines de recherche et de formation, a pour objectif :

- la production de recherches de qualité qui auront un impact sur les politiques de santé ;

- la mise à disposition de scientifiques et d'équipes de recherche compétitifs ;

- le renforcement du management de la recherche ;

- l'amélioration de l'environnement de la recherche.

Ces collaborations entre les institutions de formation et de recherche, les différents ministères impliqués, les agences de développement, les organisations non gouvernementales et le secteur privé ont permis l'obtention de progrès significatifs dans la lutte contre les endémies parasitaires. Des travaux de recherche ont apporté des connaissances nouvelles et/ou fourni des résultats prometteurs : le rôle de l'ivermectine sur la transmission du paludisme, l'évaluation des triples thérapies sur les MTN (praziquantel, ivermectine et albendazole) ou la mise en évidence des formes hybrides de Schistosoma heamatobium et Schistosoma bovis. D'autres évaluations en cours portent sur la recherche de plateformes alternatives de distribution des traitements de masse, sur la fréquence, la couverture et la durée du traitement, sur la recherche de nouvelles techniques de diagnostic et sur l'efficacité vaccinale du Bilhvax dans l'infection bilharzienne.

Malgré ces avancées, certaines parasitoses restent prévalentes en Afrique subsaharienne. La résistance aux antihelminthiques, la faiblesse du système de surveillance, le défaut de financement et le défi sécuritaire dans certaines zones constituent des entraves à l'objectif d'élimination. Une volonté politique mieux affirmée est nécessaire ; c'est ainsi que l'Union africaine a inscrit la recherche et la formation comme une de ses priorités. Plusieurs initiatives sont prises parmi lesquelles la célébration de la Journée de la renaissance scientifique africaine le 28 juin de chaque année, la mise en place d'académies des sciences présentes au niveau de plusieurs pays, le Fonds de recherche du CAMES pour la recherche, etc.

L'Afrique est soutenue dans sa vision d'éliminer les maladies parasitaires par un large partenariat international qui appuie le renforcement des capacités ; ce sont, notamment, le partenariat européen de renforcement de capacités en recherche clinique (EDTCP), l'Agence française de développement (AFD), le Wellcome Trust, la coopération britan- nique (DFID), le NIH, la fondation Bill-et-Melinda-Gates, la Banque islamique de développement, la Banque mondiale, etc. Ces partenariats ont permis la création de centres d'excellence africains de recherche et de formation avec, déjà, 58 centres opérationnels dans 45 universités. Le partenariat présent à l'intérieur des sociétés scientifiques comme la Société africaine de parasitologie (SoAP) et la Société de l'initiative multilatérale sur le paludisme (MIM Society) participe au maintien de l'excellence de la recherche et de la formation, indispensable pour éliminer les endémies parasitaires du continent africain.

\section{Le paludisme aujourd'hui et son traitement}

\section{Danis}

Professeur émérite, CHU Pitié-Salpêtrière, 47 bld de l'hôpital, F-75013 Paris ; Académie nationale de médecine, 16, rue Bonaparte, F-75006 Paris, France

martindanis21@gmail.com

\section{Introduction}

La lutte contre l'endémie palustre dans le monde a été remarquablement efficace entre 2008 et 2015 avec une diminution du nombre de cas et de morts comptabilisés dans les « Rapport sur le paludisme dans le monde " publiés tous les ans par l'OMS (40\% de morts en moins entre 2000 et 2011). La distribution massive de moustiquaires imprégnées d'insecticides à longue durée d'action (MILDA), la mise à disposition progressivement croissante de combinaisons thérapeutiques à base d'artémisinine (CTA) et de tests de diagnostic rapide (TDR) ont contribué à ce succès. Une mobilisation des acteurs grâce au partenariat avec Roll Back Malaria (Faire reculer le paludisme), la création de programmes nationaux de lutte contre le paludisme (PNLP), un investissement substantiel par la Banque mondiale, par le Fonds mondial, des fondations et $\mathrm{ONG}$ ont permis de financer cette lutte. Mais le rapport 2018 de l'OMS signale pour la deuxième année consécutive une dégradation de la situation (https://www.who.int/malaria/publications/world-malariareport-2018/report/fr/). Le nombre de cas dans le monde est estimé à 219 millions en 2017 (217 millions en 2016). Les dix pays d'Afrique les plus durement touchés par la maladie auraient enregistré 3,5 millions de cas de paludisme supplémentaires par rapport à 2016. Le nombre de décès dus au paludisme a été estimé en 2018 à 435000 , contre 451000 en 2016. Les enfants de moins de cinq ans ont représenté $61 \%(266000)$ des décès. À elle seule, la région Afrique de l'OMS a enregistré $93 \%$ des décès. Cependant, le paludisme ne sévit plus depuis au moins trois ans en Argentine, au Paraguay (élimination en 2018), en Géorgie, en Turquie, au Sri Lanka (élimination en 2016), dans le 
Maghreb (Algérie : 2016). La Chine déclare n'avoir observé aucun cas autochtone en 2017.

Les investissements dans le contrôle et l'élimination du paludisme ont été en 2017 de 3,1 milliards de dollars américains, dont 2,2 milliards pour la région Afrique de l'OMS. Le Fonds mondial contribue pour 1,3 milliard. Même si ce financement est relativement stable depuis 2010, il est loin d'atteindre le niveau requis pour réduire d'au moins $40 \%$ l'incidence du paludisme et la mortalité associée au plan mondial par rapport à 2015. Un financement efficace devrait s'élever à au moins 6,6 milliards de dollars américains par an d'ici 2020.

La mise à disposition des armes de la lutte antipaludique augmente :

- moustiquaires : 624 millions de MILDA livrées entre 2015 et 2017, la majorité en Afrique subsaharienne, $85 \%$ ont été distribuées gratuitement par le biais de campagnes de distribution de masse ;

- tests de diagnostic rapide : en 2017, 245 millions de TDR ont été distribués par les PNLP ; traitements : entre 2010 et 2017, les pays ont acheté 2,74 milliards de traitements par CTA, dont 1,45 milliard ont été distribués par les PNLP, et 1,42 milliard (98\%) dans la région Afrique de l'OMS.

\section{Traitements : les combinaisons thérapeutiques à base d'artémisinine}

Le traitement en trois jours du paludisme à Plasmodium falciparum non compliqué : il associe, dans un même comprimé, un dérivé semi-synthétique de l'artémisinine - activité rapide, intense, mais demi-vie courte - à un antipaludique ayant une cible d'action différente (diminue le risque de résistance) et une demi-vie longue. Cinq CTA sont disponibles :

- artéméther-luméfantrine : deux prises par jour + collation, gamétocytocide partiel, bien toléré ;

- artésunate-amodiaquine : une prise par jour, inactif sur gamétocyte, nausées parfois ;

- artésunate-méfloquine : une prise par jour, gamétocytocide partiel, troubles neuropsychos parfois ;

- dihydroartémisinine-pipéraquine : une prise par jour, à jeun, non gamétocyte, prévient longtemps les réinfections, allongement de l'espace QT parfois ;

- artésunate-sulfadoxine + pyriméthamine (SP) : comprimés séparés, résistance à $\mathrm{SP}$ fréquente ;

- partiellement disponible : artésunate-pyronaridine, transaminases à surveiller.

Diffusion : les données collectées à partir de 18 enquêtes nationales menées en Afrique subsaharienne montrent que
$88 \%$ des enfants fiévreux ayant sollicité un traitement antipaludique dans le secteur public ont reçu un traitement par CTA.

Résistance : en Afrique, aucune résistance aux artémisinines n'a été rapportée à ce jour, et les CTA de première ligne restent efficaces dans toutes les zones d'endémie palustre. Cinq études contrôlées récentes en Afrique centrale, enfants de moins de cinq ans suivis de j28 à j42, parasitémies PCR ajustées, montrent 93 à $99 \%$ de guérisons ; une étude génomique : absence de marqueur moléculaire de résistance dans six régions de République démocratique du Congo (Mvumbi DM, PloS One 2017).

Chimiorésistance de P. falciparum aux CTA dans la sousrégion du "Grand Mékong » : le nombre de CTA ayant un taux élevé d'échec : Cambodge : 4 ; Thaïlande : 3 ; Vietnam : 2 ; Laos, Myanmar et Yunnan (Chine) : 1 .

La multirésistance, qui inclut la résistance (partielle) aux artémisinines et aux médicaments partenaires, a été détectée dans ces cinq pays.

Fréquence de la mutation K13, marqueur de la résistance et identification des allèles en cause. Programme pour circonscrire la zone, empêcher la diffusion et éliminer P. falciparum.

On a cependant pu observer une réduction massive du nombre de cas de paludisme et de décès associés dans cette sous-région.

\section{Stratégies thérapeutiques : du contrôle à l'élimination}

\section{Paludisme à P. falciparum grave et compliqué}

Les populations concernées sont, en zone de transmission forte et pérenne, les enfants de quatre mois à quatre ans, en zone de transmission saisonnière, ceux de quatre mois à dix ans et, en zone de faible transmission localisée ainsi que pour les voyageurs, à tout âge.

Traitements : avant 2005 : quinine i.v. ; létalité 20 à $30 \%$ (France $\leq 10 \%$ ).

Essais contrôlés déterminants :

- 2005 : Asie, adultes artésunate i.v. versus quinine i.v. (Seaquamat, Dondorp MD Lancet 2005) : réduction de $35 \%$ de la mortalité dans le bras artésunate ;

- 2010 : Afrique, enfants artésunate i.v. versus quinine i.v. (Aquamat, Dondorp MD Lancet 2010) : réduction de $22,5 \%$ de la mortalité dans le bras artésunate ;

- 2011-2016 : en France, artésunate i.v. versus quinine (Elket N, Clin Infect Dis 2019) : mortalité quinine : $4 \%$, artésunate : $3 \%$; dans le bras artésunate, le patient quitte l'unité de réanimation plus vite, mais un effet indésirable est observé, $15 \%$ d'hémolyses retardées, non observé chez les enfants en Afrique. 


\section{Traitement antipaludique intermittent des femmes enceintes}

Administration systématique lors des consultations prénatales de sulfadoxine-pyriméthamine (SP), protection efficace de la femme et du foetus des conséquences du paludisme gestationnel (Diakite OS, Doumbo OK Clin Infect Dis. 2011) ; sur 33 pays africains ayant communiqué des données de couverture en traitement antipaludique intermittent des femmes enceintes (TPIp) par SP en 2017, seulement $22 \%$ des femmes enceintes éligibles avaient reçu trois doses de TPIp (comme recommandé par l'OMS), contre $17 \%$ en 2015 et $0 \%$ en 2010.

\section{Chimioprévention du paludisme saisonnier}

(Noor AM, Doumbo OK, Snow RW. PLoS One 2015) : En 2017, 15,7 millions d'enfants vivant dans 12 pays d'Afrique sahélienne ont été protégés par des programmes de chimioprévention du paludisme saisonnier (CPS). Cependant, quelque 13,6 millions d'enfants qui auraient pu bénéficier de cette intervention n'ont pas été couverts, principalement à cause d'un manque de financements.

\section{Nouveaux antipaludiques en développement}

Une dizaine de molécules identifiées, mais trois ou quatre seulement en phase 2, voir « Medicines for Malaria Venture » (https://www.mmv.org).

- AQ-13 : amino-4-quinoléine : + chaîne latérale, actif sur chloroquine R, phase 2 ;

- DSM265 : triazolopyrimidine, inhibiteur de Plasmodial dihydroorotate dehydrogenase (DHOR);

- KAE609 : spiroindolone, cipargamin, phase 2 ;

- KAF156 : imidazolopipérazine : phase 2, associé à luméfantrine, chimiorésistance obtenue in vitro ;

- OZ277 : ozonide, artérolane : risque de résistance croisée avec artémisinine?

- OZ439 : ozonide, artéfénomel : phase 2, associé à ferroquine ;

- DDD107498 : quinoline-4-carboxamide : cible, inhibition du translation elongation factor 2 (PfEF2);

- MMV048 : 2-aminopyridine : cible, phosphatidylinositol 4-kinase ;

- UCT943 : 2-aminopyridine, optimisé MMV048 ;

- SJ733 : dihydroisoquinolone : cible : PfATP4 ;

- Artemisia annua : autres composés que l'artémisinine : flavonoïdes, terpénoïdes?

- Artemisia afra : guaianolide sesquiterpène lactones, $1 \alpha, 4 \alpha-$ dihydroxybishopsolicepolide, yomogiartemin, gamétocytocide?
- Aglaia genus : rocaglates : CR-1-31B, cible : initiation factor 4A (eIF4A) protein synthesis, neuropaludisme?

Objectif : identifier un composé ayant une cible différente de celle des médicaments actuels, actif sur tous les stades du cycle des plasmodies : hypnozoïte, sporozoïte, trophozoïte, gamétocyte, actif en une prise, un seul jour ! Associé à un ou deux autres antipaludiques à mode d'action différent et pharmacocinétique longue. Un rêve !

\section{Une nouvelle cible dans la lutte antipaludique}

Les porteurs asymptomatiques persistants de $P$. falciparum ou Plasmodium vivax indétectables par les techniques standard, microscopiques ou TDR. Dans les zones de faible transmission, saisonnière en Afrique, la persistance dans une petite partie de la population, pendant toute la saison sèche, d'un cycle érythrocytaire, sans signe clinique, à parasitémie submicroscopique avec présence de gamétocytes, est responsable de la reprise épidémique dès le début de la saison des pluies.

Exemple de deux études :

- Namibie : étude de 1919 prélèvements par RDT et amplification moléculaire isothermale (LAMP) : 0,8 \% par RDT et 2,2 \% par LAMP (McCreesh P. Malar J 2018) ;

- Sénégal : prélèvements avant la saison des pluies trois années consécutives chez 612 à 723 sujets asymptomatiques testés par microscopie et par PCRq : Plasmodium positif en PCR 3,75, 12,44, 6,41 \% avec $P$. falciparum dans 81 à $95 \%$ des cas (Niang M, Touré-Baldé A. PLoS One 2017).

Conclusion : les stratégies d'élimination du paludisme doivent envisager l'utilisation de méthodes de diagnostic moléculaires pour identifier les porteurs asymptomatiques de plasmodies et décider du protocole : traitement de masse ou ciblé, médicament gamétocytocide.

\section{Administration d'un traitement de masse (MDA) en Asie du Sud-Est}

Impact sur l'élimination de $P$. falciparum d'un traitement de masse ciblé par DHA-pipéraquine dans 16 villages concernés par la résistance partielle à l'artémisinine : comparaison entre huit villages traités aux mois M0, M1, M2 et huit villages au mois M12 : baisse importante de la prévalence et de l'incidence au mois 3 dans les huit villages traités, mais reprise progressive de la transmission au mois 12. Difficultés liées à l'hétérogénéité des villages et aux mouvements de population (von Seidlen L, Nosten FH, Dondorp AM, White NJ. et al PloS Med 2019). 


\section{Conclusion}

Au total, la lutte pour le contrôle du paludisme a marqué le pas depuis deux ans, en particulier en Afrique subsaharienne, sans doute en raison d'un financement insuffisant, mais aussi de désorganisation des systèmes de santé et de difficultés logistiques liées aux conflits sociopolitiques. La diffusion d'antipaludiques falsifiés joue aussi un rôle croissant.

Dire que les tisanes de feuilles sèches d'Artemisia seraient la solution à l'élimination est une illusion dangereuse. Mieux vaut œuvrer pour la disponibilité jusque dans les villages du CTA recommandé : l'agent de santé communautaire, désigné par les villageois, doit être formé, savoir plaider pour l'utilisation des moustiquaires, disposer de TDR et de CTA.

« Réduire le paludisme là où il pèse le plus ».

\section{Les vecteurs et la place de l'entomologie dans la lutte contre le paludisme}

\section{F. Simard}

MIVEGEC (Maladies infectieuses et vecteurs : écologie, génétique, évolution et contrôle), UMR IRD-CNRSuniversité de Montpellier, Montpellier, France

frederic.simard@ird.fr

Le paludisme-maladie est provoqué par l'infection puis le développement chez l'homme d'un parasite du genre Plasmodium généralement transmis par la piqûre d'un moustique vecteur. Si la vision médicale du paludisme met volontiers l'homme - que l'on cherche à protéger ou à guérir de l'infection - au centre du processus, biologiquement parlant, l'homme n'est qu'un hôte particulier, un « environnement », au sein duquel le parasite effectue une partie seulement de son cycle de développement. D'autres hôtes, mammifères, oiseaux ou reptiles hébergent également diverses espèces de Plasmodium sans pour autant développer de symptômes cliniques aussi graves que ceux produits chez l'homme par une infection à Plasmodium falciparum.

L'insecte vecteur, par contre, est l'hôte définitif du parasite, puisque c'est chez le moustique qu'a lieu la reproduction du Plasmodium, c'est dans son estomac que se passe la fécondation, étape clé de la biologie de tout être vivant à reproduction sexuée. C'est également le moustique qui fait le lien entre environnement et transmission des Plasmodium et c'est via ce filtre que se façonnent la dynamique et l'intensité de la transmission des Plasmodium, et leur évolution dans le temps et dans l'espace. Ainsi en Afrique, berceau du paludisme comme de l'Humanité et continent qui continue à payer le plus lourd tribut à la maladie, plusieurs espèces d'anophèles peuvent transmettre les Plasmodium en même temps au même endroit, où se relayer au cours des saisons, donnant lieu à divers faciès épidémiologiques de transmission et diverses manifestations de la maladie.

Bien comprendre la biologie et l'écologie des vecteurs, identifier les paramètres biotiques et abiotiques du milieu qui en régissent la distribution géographique, la diffusion, la survie, étudier les mécanismes génétiques et non génétiques impliqués dans les interactions qu'entretiennent les moustiques non seulement avec les Plasmodium qu'ils transmettent, mais également avec les diverses composantes des écosystèmes au sein desquels ils se développent deviennent alors nécessaires pour mieux comprendre l'épidémiologie de la transmission du paludisme et ses hétérogénéités spatiales et temporelles. Replacer les vecteurs au cœur du système pathogène permet en outre d'envisager une autre cible que l'agent infectieux lui-même pour lutter contre la propagation de la maladie et donne toute sa pertinence et sa légitimité à la lutte antivectorielle pour le contrôle du paludisme. C'est là toute la place de l'entomologie médicale dans la recherche sur et dans la lutte contre le paludisme.

Et les succès sont au rendez-vous. Une étude récente a montré qu'entre 2000 et 2015 la prévalence des infections à $P$. falciparum en Afrique subsaharienne a baissé de $50 \%$, et l'incidence clinique de la maladie a diminué de $40 \%$, représentant plus de 650 millions de cas cliniques évités en grande partie (68 \%) grâce au déploiement à large échelle de moustiquaires imprégnées d'insecticides. Les auteurs estiment par ailleurs que l'utilisation d'insecticides rémanents en pulvérisation intradomiciliaire, une stratégie mise en place dans plusieurs pays d'Afrique au cours des années 2000, explique $10 \%$ supplémentaires de ces cas évités, renforçant l'impact de la lutte contre les anophèles pour le contrôle du paludisme. Mais ces succès sont fragiles.

Les anophèles, vecteurs majeurs des Plasmodium en Afrique, ont tous développé des résistances aux différentes molécules qui sont aujourd'hui disponibles pour un usage en santé publique. Ces résistances sont liées à divers mécanismes génétiques (mutations dans la cible des insecticides), enzymatiques (détoxification et/ou compensation des effets toxiques des insecticides) ou comportementaux (heures et lieux de piqûre) qui menacent directement l'efficacité des méthodes de lutte aujourd'hui en place, au point que les succès conquis de haute lutte s'émoussent rapidement. Les vecteurs s'adaptent, comme ils l'ont toujours fait, aux modifications de leur environnement, et c'est toute l'épidémiologie de la transmission du paludisme qui évolue vers une « transmission résiduelle » par des moustiques qui piquent à l'extérieur, en soirée ou à l'aube, en zone urbaine polluée... Là encore, la recherche en entomologie doit permettre de trouver des solutions pour une gestion efficace et durable du risque vectoriel.

De nombreuses facettes de la biologie des vecteurs restent à exploiter, et plusieurs pistes de recherche sont actuellement 
suivies pour permettre le développement de nouvelles approches et d'outils innovants pour le contrôle des vecteurs. Des travaux sur le comportement de reproduction des moustiques, sur les stimuli qui les orientent vers un partenaire sexuel, un hôte à piquer, un site de ponte où déposer leurs œufs, sur le développement et l'écologie de leurs stades larvaires, font émerger des solutions innovantes dans de nombreux laboratoires de recherche, au Nord comme au Sud.

L'objectif de toutes ces études n'est pas d'améliorer ce qui existe, mais de proposer des méthodes et outils alternatifs de lutte antivectorielle, complémentaires et adaptables en fonction des contextes épidémiologiques, écologiques et sociétaux pour une gestion intégrée des vecteurs, en accord avec les recommandations de l'OMS. Dans ce contexte, la possibilité d'utiliser les moustiques eux-mêmes, de manipuler leur comportement ou leur capital génétique pour diffuser un principe actif au sein des populations naturelles de vecteurs est une perspective qui mobilise plusieurs groupes de recherche de par le monde. De nouvelles technologies sont à l'étude et incluent par exemple la lutte par lâchers massifs de mâles stériles, le développement de molécules attractives ou répulsives pour piéger les vecteurs, ou encore l'utilisation de moustiques transgéniques en vue d'éliminer ou de modifier durablement les populations naturelles de vecteurs de manière à les rendre incapables de transmettre les Plasmodium.

Depuis la fin des années 1990, les développements majeurs et rapides de la biologie moléculaire et de la bioinformatique ont en effet permis d'amasser de nombreuses données sur l'architecture et la composition des génomes des vecteurs et d'identifier certains gènes ayant un effet majeur sur la biologie du moustique ou sa capacité intrinsèque à permettre le développement des agents pathogènes et à les transmettre à l'homme. L'édition précise du génome à l'aide du système CRISPR-Cas9, ciseaux moléculaires de précision mis au point depuis 2012, a ainsi permis de « construire» plusieurs colonies de moustiques transgéniques qui pourraient, demain, être utilisées pour la lutte contre les moustiques et la transmission du paludisme.

L'introduction d'une mutation dans le gène doublesex impliqué dans le déterminisme du sexe chez le moustique Anopheles gambiae, vecteur majeur de $P$. falciparum en Afrique, a ainsi pour effet de bloquer l'épissage correct des introns chez les femelles (mais pas chez les mâles), produisant un phénotype « transgenre » complètement stérile, affublé d'attributs mâles et femelles (organes sexuels, antennes, etc.) et d'un appareil piqueur altéré qui ne leur permet ni de piquer ni de se reproduire. Des expériences en laboratoire ont montré que l'introduction de quelques mâles porteurs de la mutation permettait l'élimination totale d'une population de moustiques de 600 individus en 8 à 12 générations, quand cette mutation est associée à un système de forçage génétique. Le forçage génétique, ou gene drive, consiste à aug- menter l'héritabilité d'un élément génétique par rapport aux conditions naturelles (lois de Mendel). La technologie CIRSPR-Cas9 peut en effet également être utilisée en ce sens par une manipulation génétique aujourd'hui à la portée d'un nombre croissant de laboratoires de recherche. C'est ainsi qu'un autre moustique vecteur, Anopheles stephensi, qui assure la grande majorité de la transmission des Plasmodium en Inde, a été transformé génétiquement de manière à exprimer et à transmettre à sa descendance deux anticorps monoclonaux qui reconnaissent et éliminent le Plasmodium à deux stades différents de son développement dans le moustique, l'ookinète dans l'estomac et les sporozoïtes dans les glandes salivaires. Là encore, les études de laboratoire ont apporté la preuve de principe de l'efficacité de l'approche, les femelles transgéniques qui expriment les anticorps suite à un repas sanguin infecté par des gamétocytes de Plasmodium étant complètement réfractaires à son développement et incapables de retransmettre le parasite. Aidée du forçage génétique, cette capacité à résister au Plasmodium se transmet efficacement de génération en génération, jusqu'à envahir totalement les populations de moustiques testées en conditions expérimentales au laboratoire. En Afrique comme en Inde, des sites pilotes sont en préparation pour recevoir, à un horizon qui reste à déterminer, les premiers lâchers expérimentaux de ces moustiques transgéniques.

Ainsi, le futur de la lutte antivectorielle se prépare et se construit dans les laboratoires de recherche du monde entier pour préserver les acquis récents de la lutte contre le paludisme en Afrique et renforcer son impact pour la santé des populations. Mais ce ne sont pas les chercheurs qui décideront de la mise en place de l'une ou l'autre de ces nouvelles technologies et de leur déploiement sur le terrain, à échelle opérationnelle pour la lutte contre les vecteurs et la prévention du paludisme. L'ensemble des parties prenantes, décideurs, opérateurs publics et privés, financeurs et citoyens, doit s'emparer des enjeux de société, réglementaires et économiques que ces nouvelles technologies soulèvent afin de bâtir ensemble, et avec les scientifiques, un futur durable sans paludisme.

\section{Le traitement préventif intermittent contre le paludisme en Afrique chez les enfants et les femmes enceintes : succès et doutes}

F. Ntoumi

Fondation congolaise pour la recherche médicale et université Marien-Ngouabi, Brazzaville, République du Congo

Institut de médecine tropicale, université de Tübingen, Tübingen, Allemagne

ffntoumi@hotmail.com 
La femme enceinte et l'enfant représentent la population la plus vulnérable au paludisme. Afin de réduire les conséquences de cette infection telles que le faible poids de naissance ou la mortalité maternelle et infantile, l'OMS a recommandé, depuis 2012, des mesures de prévention pour ces deux cibles. Chez la femme enceinte, le traitement préventif intermittent (TPIp) se donne au cours des visites prénatales quel que soit le statut infectieux face à Plasmodium falciparum de la femme (sauf VIH positives). Pour l'enfant, les mesures préventives concernent ceux vivant en zone de transmission saisonnière du paludisme, donc en zone sahélienne, et le TPIp se donne durant trois ou quatre mois au cours de l'année (en période de transmission). Pour ces deux cibles, la combinaison thérapeutique utilisée pour le TPIp est la sulfadoxine-pyriméthamine (SP).

La moustiquaire imprégnée d'insecticide est l'outil le plus utilisé et, dans beaucoup de pays, elle est distribuée gratuitement aux femmes enceintes et pour les enfants de moins de cinq ans. Il apparaît que le TPIp-SP (traitement préventif intermittent à la sulphadoxine-pyriméthamine pour la femme enceinte) est adopté par $83 \%$ des pays endémiques d'Afrique mais inégalement utilisé. Cependant, il est noté dans le rapport de l'OMS de 2017 que les femmes qui reçoivent les première, deuxième et troisième doses représentent 54,42 et $22 \%$, respectivement.

Une publication récente analysant les données de plusieurs études dans différents pays a mis en évidence une augmentation significative de la résistance des parasites $P$. falciparum à la SP dans les pays utilisant cet antipaludique. De plus, l'efficacité de la SP pour TPIp est réduite dans les zones où $P$. falciparum est fortement résistant à la SP. Cela s'observe au niveau du poids de naissance de l'enfant.

En ce qui concerne le traitement préventif intermittent chez l'enfant (TPIc), le nombre d'enfants cibles le recevant est variable dans les 12 pays où il a été mis en œuvre. Dans certains d'entre eux, le taux est très encourageant comme au Mali, mais dans d'autres pays comme le Nigeria, le taux est encore trop faible.

Il est urgent de mettre à disposition de la femme de nouvelles molécules antipaludiques bien tolérées et accessibles. Le rapport de l'OMS de 2017 montre aussi qu'il reste un nombre significatif d'enfants qui n'ont pu bénéficier du TPIc. Les ressources humaines et financières doivent continuer à être mobilisées afin de ne pas annuler tous les efforts consentis depuis ces dernières décennies dans la réduction du poids de la maladie en Afrique.

\section{Les essais vaccinaux contre le paludisme en Afrique (résumé rédigé par J.-P. Chippaux)}

\section{O. Leroy}

Fondatrice et coordonnatrice de l'European Vaccine Initiative
Ogobara Doumbo - qui m'appelait sa grande sœur, mais dont je tiens à préciser qu'il fut mon maître - était un visionnaire et un pionnier dans son domaine. Son humanité et sa rigueur servent d'exemple aux chercheurs du monde entier.

$\mathrm{La}$ vaccination contre le paludisme constitue une ancienne préoccupation. Edmond et Étienne Sergent, dès le début du $\mathrm{XX}^{\mathrm{e}}$ siècle, ont montré l'acquisition d'une immunité protectrice contre le paludisme aviaire après l'inoculation de sporozoïtes de Plasmodium relictum à des poulets (C R Acad Sci 1910;151:407-9).

La complexité du développement d'un vaccin contre le paludisme tient à celle de son cycle biologique. Trois cibles peuvent être envisagées, qui consistent à prévenir :

- l'infection ;

- les formes graves ou le décès dus à la maladie ;

- ou la transmission du parasite.

Dans chaque cas, il convient d'identifier les antigènes pertinents afin d'induire une réponse immune protectrice et d'éviter les mécanismes d'échappement immunitaire.

De nombreux antigènes ont fait l'objet de développements plus ou moins poussés. Ils concernent les stades préérythrocytaires (du sporozoïte inoculé lors de la piqûre de l'anophèle au stage hépatique), sanguins (mérozoïte infectant le globule rouge où il devient trophozoïte responsable de la maladie) et les formes sexuées (gamétocytes mâles et femelles), également sanguines, ingérées par l'anophèle lors d'une nouvelle piqûre. Les vaccins contre les gamétocytes assureraient le blocage de la transmission.

On peut distinguer deux catégories d'essais vaccinaux : ceux qui visent à établir une preuve de concept de la capacité immunogène et protectrice de l'antigène sélectionné, et les essais permettant la validation d'un candidat vaccin, c'est-àdire confirmer son innocuité et évaluer son efficacité. En 2018, 25 sites d'essais vaccinaux contre le paludisme étaient actifs, dont 17 en Afrique subsaharienne.

L'Organisation mondiale de la santé (OMS) a défini trois objectifs au vaccin contre le paludisme :

- réduire la mortalité et l'incidence de $40 \%$ en 2020 , de $75 \%$ en 2025 et de $90 \%$ en 2030 ;

- éliminer la maladie dans 10,20 et 35 pays à transmission en 2020, 2025 et 2030 respectivement ;

- prévenir la réapparition du paludisme dans les pays où il a été éliminé.

Il s'agit là d'un programme particulièrement ambitieux... Trois stratégies sont actuellement développées :

- celle d'Adrian Hill (université d'Oxford) est fondée sur l'utilisation d'un vecteur immunologique constitué d'un poxvirus ou d'un adénovirus et combinant plusieurs types d'antigènes éventuellement administrés à des temps 
différents. Cette stratégie demeure à la phase de preuve de concept et Ogobara Doumbo n'y a pas été associé ;

- celle de Stephen Hoffman (Sanaria), qui utilise des sporozoïtes atténués (PfSPZ). Le Centre de recherche et de formation sur le paludisme (MRTC) de Bamako a fortement collaboré avec la plateforme Sanaria pour évaluer le PfSPZ. Ce dernier a fait l'objet d'une quarantaine d'essais cliniques. Cependant, la disparité des résultats est importante. Elle souligne les défis auxquels ce vaccin est confronté : paraspécificité inconnue, production complexe et coûteuse des sporozoïtes, administration par voie veineuse et conservation en azote liquide, ce qui ne simplifiera pas son utilisation s'il s'avérait efficace et bien toléré ;

- celle de Joe Cohen (GSK) qui développe le RTS,S constitué d'une protéine circumsporozoïte (protéine de surface du sporozoïte) couplée à l'antigène du vaccin contre l'hépatite B. Le RTS,S a fait l'objet de plus de 55 essais cliniques à ce jour. Une phase III menée dans dix sites représentant des modèles différents de transmission concerne 16000 jeunes enfants, la moitié de 6 à 12 semaines et le reste de 5 à 17 mois. Son objectif est d'évaluer son innocuité et son efficacité. Trois doses ont été administrées. Les résultats, sans être spectaculaires, sont prometteurs. L'efficacité clinique est de l'ordre de $30 \%$, plus élevée chez les enfants de plus de cinq mois lors de la première administration, mais de courte durée (17\% après quatre ans). D'une part, l'efficacité augmente significativement après un rappel à 20 mois ( 36,3 versus $28,3 \%$ ). D'autre part, le vaccin permet d'éviter un grand nombre de cas de paludisme clinique, davantage chez les enfants de 5 à 17 mois que chez les nourrissons de moins de 12 semaines au moment de la première dose de vaccin. Cependant, une augmentation de l'incidence des méningites chez les enfants vaccinés a été observée, ce qui nécessite des investigations supplémentaires (Greenwood et Dumbo (2016) Lancet 387:318-9). Quoi qu'il en soit, il subsiste quelques réserves sur l'efficacité protectrice et l'innocuité du RTS,S.

En conséquence, l'OMS a recommandé de poursuivre les études pilotes dans différents faciès épidémiologiques. Le nombre de doses administrées est de trois injections plus un rappel à 20 mois, afin d'évaluer :

- la faisabilité de ce schéma vaccinal ;

- l'impact du vaccin sur la mortalité infantile ;

- la tolérance du vaccin, notamment le risque d'incidence des méningites et du paludisme cérébral ;

- l'impact de la stratégie vaccinale antipalustre sur les autres programmes de vaccination et méthodes de prévention du paludisme.

Il serait, en outre, intéressant de s'assurer de l'absence de surmortalité féminine contrairement à ce qui a été rapporté au cours de l'étude précédente.
L'apport du MRTC dans les essais de vaccins contre le paludisme a été essentiel. Grâce à sa ténacité et à son sens de l'organisation, Ogobara Doumbo a permis l'implantation au Mali de six sites de recherche clinique permanents, dont cinq dédiés aux essais cliniques contre le paludisme. Pas moins de 18 vaccins contre les stades pré-érythrocytaires, sanguins, bloquant la transmission ou même contre le paludisme gestationnel - utilisant divers adjuvants - y ont été étudiés, en collaboration avec plusieurs institutions ou laboratoires pharmaceutiques. De plus, il a conduit trois études d'infection humaine contrôlée du paludisme, outil décisif pour évaluer la protection d'un candidat vaccin.

Au-delà de ces résultats remarquables, le rôle d'Ogobara Doumbo dans l'organisation du MRCT, tant au niveau institutionnel qu'individuel, a été déterminant. Il était attaché, notamment, à la promotion des chercheurs, tout particulièrement celle des femmes qu'il a toujours encouragées et soutenues.

\section{L'éradication du paludisme : un défi pour 2050}

\section{A. Massougbodji}

Professeur honoraire de parasitologie, directeur de l'Institut de recherche clinique du Bénin, Abomey-Calavi, Bénin massougbodjiachille@yahoo.fr

Le contrôle, l'élimination et l'éradication constituent un même combat contre une maladie, mais révèlent des ambitions différentes, ce qui n'est pas forcément évident, particulièrement dans les documents rédigés en anglais (comme on sait, la langue de ceux qui dictent les agendas mondiaux). Le contrôle d'une maladie consiste à en réduire le fardeau sans intention d'en supprimer les causes. L'élimination vise à interrompre localement la transmission. L'éradication entend supprimer définitivement la maladie à l'échelle mondiale. C'est de cette dernière dont il est question d'ici à 2050.

L'histoire de la lutte contre le paludisme est une succession d'espoirs et de déconvenues. Dès 1950, l'éradication du paludisme était envisagée. Cet objectif a été poursuivi jusqu'en 1970, date à laquelle, en raison de la complexité du projet et d'échecs avérés, on est revenu à davantage de modestie.

L'espoir de réussite se fondait sur quelques outils qui semblaient décisifs. Au premier rang venait le DDT qui avait fait ses preuves pendant la Seconde Guerre mondiale, spectaculairement efficace alors que l'on se préoccupait peu de préservation des écosystèmes et de l'environnement. Il y avait également un corps de santé, composé d'excellents cliniciens et de biologistes, notamment de microscopistes performants, bien formés au diagnostic. Les antimalariques étaient alors efficaces, simples d'utilisation et peu coûteux : quinine, amino-4-quinoléines (chloroquine en particulier) et 
sulfadoxine-pyriméthamine pour les principaux. Plusieurs stratégies ont été mises en œuvre simultanément afin de mutualiser leur efficacité propre : chloroquinisation de masse à visée préventive en milieu scolaire, traitement des cas aussi précocement que possible, démoustication par pulvérisations de DDT intra- et extradomiciliaires, hygiène du milieu pour réduire le risque de reproduction des moustiques. Le plus important, sans doute, fut la volonté politique ferme, permanente et la discipline au point de conduire, parfois, à une certaine forme de coercition. Cette volonté politique se traduisait par une mobilisation institutionnelle remarquable avec l'appui des gouvernements coloniaux, puis nationaux, et la mise en place d'un service de santé puissant bénéficiant d'un financement autonome : à l'époque, ne parlait-on pas à propos des " Grandes Endémies » d'un État dans l'État? Cela a favorisé une motivation communautaire forte, élément fondamental à la réussite de toute entreprise de cette nature.

Alors que l'on pensait tous les atouts réunis pour conduire au succès, le résultat fut un échec retentissant et décourageant. L'apparition rapide des résistances - anophèles au DDT puis aux autres insecticides proposés en remplacement, d'une part, et plasmodiums aux antimalariques, d'autre part a imposé une révision à la baisse des ambitions premières. L'objectif d'éradication s'est transformé en contrôle du paludisme, non sans quelques irréductibles qui continuaient d'envisager une élimination par endroits et sous certaines conditions... Si le contrôle reste un but minimal, l'élimination ne doit pas être considérée comme un glissement sémantique mais correspondrait à un phénomène volontariste de suggestion : si l'on «peut » l'élimination, pourquoi pas l'éradication ? L'intérêt d'un plaidoyer raisonnable est qu'il séduit davantage, d'autant plus que, depuis le fâcheux constat d'échec, de nouveaux outils et paradigmes sont apparus. Cependant, bien que prometteurs, ils font apparaitre d'autres contraintes ou limites.

La lutte antivectorielle a considérablement évolué comme l'a rappelé si justement Frédéric Simard dans sa présentation. Les insecticides - et leur utilisation - se sont profondément diversifiés, avec une efficacité et des modes d'emploi plus ciblés, ce qui n'empêche pas la surveillance de leur impact sur l'environnement qui reste une préoccupation majeure. Les moyens diagnostiques ont eux aussi connus des changements remarquables. À côté de tests de diagnostic rapide qui ne nécessitent plus de compétence technique spécifique, les aspirations des cliniciens et des biologistes se sont élargies à des activités de gestion, supervision, formation, au risque de les éloigner du patient. L'arsenal thérapeutique, en revanche, a peu changé. Ce sont, dans l'ensemble, les mêmes antimalariques, avec des présentations parfois nouvelles, désormais prescrits en association. Les dérivés de l'artémisinine (ACT), produit très ancien mais récemment promu dans la pharmacopée occidentale, doivent eux aussi faire face aux résistances des plasmodiums même si elles ne sont pas encore décrites en Afrique. Plus qu'auparavant, nous sommes envahis par les faux médicaments et confrontés aux mésusages, de plus en plus fréquents, des médicaments en général et des antimalariques en particulier. Il suffit de voir les offres de tisanes à base de feuilles d'Artemisia qui encombrent les réseaux sociaux - et même qui apparaissent dans la littérature médicale et scientifique - pour comprendre qu'un nouveau front s'est ouvert et, sans doute, pas des plus simples! Il nécessitera une réponse complexe, car ces tisanes bénéficient d'un fort potentiel de sympathie. Les stratégies se sont également adaptées. Elles ciblent plus précisément des groupes à risque, notamment les populations les plus vulnérables, comme les enfants et les femmes enceintes, ou des faciès écologiques associés à la biologie des vecteurs ou aux modes de transmission du plasmodium, pour renforcer l'efficience des méthodes de lutte. La prise en charge domiciliaire des cas (PECADOM) est mise en œuvre largement dans de nombreux pays subsahariens afin de réduire la morbidité et la mortalité, notamment chez les jeunes enfants. L'utilisation de moustiquaires imprégnées d'insecticides à longue durée d'action (ou l'application d'insecticides sur d'autres supports comme les rideaux, voire la pulvérisation intradomiciliaire) vise à empêcher la transmission du parasite. Tout cela doit évidemment être accompagné d'une surveillance épidémiologique et entomologique qui permettra l'évaluation de l'impact - positif ou négatif - de la stratégie. Cependant, la volonté politique s'est essoufflée. Les déclarations institutionnelles sont ambiguës, dans un souci de considérer l'ensemble des aspirations de populations attentives - peut-être davantage qu'auparavant - à leur liberté. Si la mobilisation communautaire reste potentiellement forte, la population dans son ensemble ne se soumet plus à l'autorité administrative ou politique et peut même s'y opposer vigoureusement.

Tout programme de lutte qu'il s'agisse de contrôle, d'élimination ou d'éradication coûte cher et a besoin de ressources humaines, techniques et financières. Si à l'époque coloniale elles ne manquaient pas, pour des raisons à la fois politiques et circonstancielles, l'indépendance a induit un changement de priorité pour satisfaire des dépenses de souveraineté et donc un relâchement pour les budgets de la santé ou de l'éducation. Dans ce contexte, la lutte contre le paludisme a rapidement perdu ses ressources nationales. Les financements extérieurs - dont on connaît la précarité et surtout les contraintes - sont rapidement devenus majoritaires. Roll Back Malaria, en 1996, allait donner un formidable sursaut à la lutte contre le paludisme, brusquement redécouvert comme un redoutable assassin responsable de freiner le développement. On assiste alors à une progression vertigineuse des financements qui ont été multipliés par 20 entre 2000 et 2015, rien que pour le soutien de la Banque mondiale, et ont permis, selon l'OMS, de réduire l'incidence de 
$30 \%$ et la mortalité de $50 \%$ (OMS, 2015. Stratégie technique mondiale de lutte contre le paludisme. 2016-2030, Genève). Dans le même temps, les ressources financières se sont diversifiées, avec une multiplication des canaux permettant une augmentation des engagements financiers de la part des organismes internationaux, de la coopération bi- et multilatérale, des ONG, des fondations d'entreprises, y compris au niveau national et gouvernemental, même si ces derniers restent marginaux. Ces financements concernent l'ensemble des postes budgétaires des programmes de lutte contre le paludisme et même au-delà puisque $14 \%$ des ressources sont affectées au renforcement du système de santé.

Il subsiste néanmoins quelques défis à relever. La gestion de la chaîne d'approvisionnement en produits et équipements n'est pas complètement maîtrisée dans la plupart des pays subsahariens, ce qui se traduit par des failles dans leur disponibilité sur le terrain. La réalisation d'activités complémentaires, notamment surveillance et évaluation des opérations, manque encore des ressources et capacités nécessaires. Les ACT tardent à être introduits et utilisés dans de nombreux pays. La supervision et l'évaluation des activités sont encore très insuffisantes. Cependant, la principale difficulté provient des financements et de leur emploi. Bien que très importants, ils restent insuffisants par rapport aux besoins réels. L'effort considérable de la communauté internationale ne saurait dispenser les États de leur responsabilité sociale vis-à-vis de leur population et, donc, de leur contribution qui doit compléter les ressources extérieures et, à terme, s'y substituer. Les mécanismes de financement doivent également être revus. Ils ne considèrent pas l'intégralité de la stratégie nationale, surtout lorsqu'elle est intégrée, et créent dans l'intervalle entre deux projets une préjudiciable discontinuité des activités. Les ressources extérieures déresponsabilisent de fait les structures publiques et accentuent le sentiment d'éparpillement géographique et institutionnel des interventions de la lutte antipaludique. Plus grave, elles sont souvent responsables du siphonnage des compétences nationales, avec le manque de capacité des structures publiques et un affaiblissement du plaidoyer. On comprend que la menace de la perte du leadership national pèse sur la pérennisation des activités et des résultats. Enfin, il faut garder présent à l'esprit que les phases ultimes de tout processus d'éradication nécessitent des efforts disproportionnés aux résultats particulièrement modestes. Du fait de la loi des rendements décroissants, cela représente un facteur de découragement pour les bailleurs, comme pour l'ensemble des acteurs. On sait également qu'un relâchement à ce stade s'avère désastreux, comme ce fut le cas pour la trypanosomose humaine africaine et aujourd'hui pour le paludisme au Venezuela.

À l'image de réussites incontestables, naguère la variole et maintenant la dracunculose, l'éradication est un objectif crédible. Il est, en tout cas, plus séduisant que le contrôle ou l'élimination, car il porte en lui l'espoir de la fin d'un fléau et, surtout, de l'arrêt des dépenses engagées pour la lutte. Pour autant, est-on sûr de disposer des bons outils et des bonnes stratégies pour y parvenir ? Peut-on affirmer que la volonté politique et sociale d'éradiquer le paludisme est également partagée par tous les acteurs? Est-on capable de réussir avant 2050, voire 2100 ?

Ogo le croyait. À moins qu'il n'ait, par souci d'efficacité, entretenu l'espoir d'une possible éradication suivant la devise de Guillaume d'Orange, «Point n'est besoin d'espérer pour entreprendre ni de réussir pour persévérer »!

Mais Ogo avait l'art de convaincre...

\section{Clôture de la journée d'hommage à Ogobara Doumbo}

\section{P. Auger}

Président du Coped, membre de l'Académie des sciences, 23, quai de Conti, F-75006 Paris, France

pierre.auger@ird.fr

Le Comité des pays en développement - le Coped - que j'ai l'honneur de présider aux côtés de François Gros, président honoraire, est très honoré d'avoir été impliqué dans l'organisation de cette journée d'hommage au Pr Ogobara Doumbo.

Je voudrais remercier l'Institut de France et tout particulièrement M. le chancelier honoraire de l'Institut de France qui nous a accueillis dans ces somptueux locaux de la Fondation Simone et Cino Del Ducca qu'il préside. Je voudrais également remercier très fortement l'Académie des sciences et l'Académie de médecine pour le parrainage et le soutien qu'elles ont bien voulu accorder à cette journée d'hommage au Pr Ogobara Doumbo.

Je remercie très chaleureusement mes confrères, François Gros et Ghislain de Marsily, pour le rôle déterminant qu'ils ont joué au sein de l'Académie et du Coped pour concrétiser la tenue de cette journée d'hommage et de colloque consacré au Pr Ogobara Doumbo. Mes remerciements s'adressent également à Mme Pascale Cossart, secrétaire perpétuelle, et au président de l'Académie des sciences, Pierre Corvol, pour leurs soutiens et leurs participations à cette journée.

Je voudrais également associer à mes remerciements Bernard Surugue, ami de longue date du Pr Ogobara Doumbo, qui a suggéré cet hommage au Coped, sans oublier JeanPhilippe Chippaux qui a pris une part très active à l'organisation scientifique de cette journée.

Je voudrais remercier tout particulièrement $M$. Alain Mérieux, président de la Fondation Mérieux, pour le support absolument déterminant qu'il a apporté très généreusement à cette manifestation.

Mes remerciements vont également au présidentdirecteur général de l'IRD, M. Jean-Paul Moatti, ainsi qu'à 
Mme Elisabeth Barbier, directrice générale déléguée, pour votre participation et votre soutien.

Je remercie tous les orateurs, les invités et les participants, tout particulièrement ceux qui sont venus de loin et notamment d'Afrique pour avoir fait le déplacement à Paris.

Je remercie Anastasia Gestkoff, Isabelle Thomas, Aurore Lopez, Daniel Malbert et Abdoulaye Ndao pour leur travail considérable au sein du Coped et de l'Académie des sciences. Merci pour votre aide précieuse dans la préparation et l'organisation de cette journée, depuis plusieurs mois.

L'hommage au Pr Ogobara Doumbo a été très émouvant, tout particulièrement avec les témoignages des membres de sa famille et de ceux qui l'ont bien connu en ayant travaillé à ses côtés, et surtout avec son message immortalisé par la vidéo qui nous a été présentée.

Les exposés de l'après-midi ont été d'excellent niveau scientifique. Ils ont permis de faire le point sur la lutte contre le paludisme et les autres maladies infectieuses en Afrique, en mettant en avant les progrès déjà réalisés et l'ampleur de la tâche restant à accomplir. Les débats et les discussions de grande qualité ont été très mobilisateurs pour que l'œuvre d'Ogobara Doumbo se poursuive et se développe dans toute sa puissance exemplaire au bénéfice des populations africaines.

Je voudrais ajouter quelques mots sur le cadre opérationnel de cet événement : le Comité pour les pays en développement. Il a été créé en 1997 par François Gros au sein de la Délégation aux relations internationales de l'Académie des sciences dans le but de promouvoir le partenariat scientifique avec les pays en développement.

François Gros en a assuré la présidence depuis l'origine jusqu'à juin 2017, ce qui représente plus de 20 ans de collaboration très fructueuse entre l'Académie des sciences et les pays en développement. Je voudrais rendre un hommage appuyé à l'action remarquable du président François Gros à la présidence du Coped, qui a permis d'élever le Coped au niveau qu'on lui connaît aujourd'hui. J'en assure maintenant la présidence à ses côtés.

Le Coped constitue un groupe permanent de réflexion, d'information et d'action concernant les enjeux scientifiques dans les pays en développement. Il accueille et soutient des forums sur des questions fondamentales pour les pays en développement, telles que les problématiques liées à la nutrition, à la gestion de l'eau ou à la prévention des maladies infectieuses, au changement climatique et à la biodiversité.

L'épidémiologie et la lutte contre les maladies infectieuses en Afrique constituent un axe scientifique stratégique primordial au sein du Coped. Cette journée en hommage à Ogobara Doumbo nous incite à poursuivre nos actions conjointes sur le chemin qu'il a tracé et appellera sans nul doute des actions futures du Coped dans cette direction.

\section{Conclusion du colloque en hommage à Ogobara Doumbo}

\section{F. Gros}

Président honoraire du Coped et secrétaire perpétuel honoraire de l'Académie des sciences, 23, quai de Conti, F-75006 Paris, France

\section{francois.gros@academie-sciences.fr}

M. le chancelier honoraire de l'Institut de France, qui nous accueillez aujourd'hui !

MM. les présidents et présidents honoraires de l'Académie des sciences, de l'Académie nationale de médecine, de l'Académie des sciences du Mali, MM. les ministres et anciens ministres de la Santé du Mali et d'Éthiopie, Mme la secrétaire perpétuelle.

M. le président-directeur général de l'IRD.

Éminentes personnalités du monde universitaire africain et de l'institut Pasteur de Lille,

M. le président de la Fondation Mérieux...

Et vous toutes et tous, membres ou proches de la famille Doumbo, qui avez bien voulu nous rejoindre et vers qui va toute notre sympathie et enfin,

Vous, cher Pierre Auger, qui venez de vous exprimer en votre qualité de président du Coped,

Chères consœurs, cher confrères, mesdames, messieurs,

Ce rappel des personnalités qui nous ont rejoints pour cet hommage solennel au Pr Doumbo, sans doute incomplet, n'en illustre pas moins le souhait, partagé par tous, d'exprimer à nouveau nos sentiments et nos souvenirs, où s'entremêlent l'admiration, l'affection et la tristesse, en pensant à l'homme exceptionnel, qui nous a quittés.

Que pourrais-je ajouter aux multiples témoignages qui ont été apportés aujourd'hui dans les très belles présentations que nous avons entendues sur la personnalité et l'œuvre du Pr Doumbo ? Un homme qui, par ses connaissances scientifiques et médicales, son émouvant et continuel souci des autres, son talent d'organisateur et de bâtisseur, dans la formidable entreprise que fut, parmi d'autres, et que demeure, le MRTC, ce centre d'excellence dans la lutte contre le paludisme. Nous souhaitons courage et succès au nouveau directeur, le Pr Djimdé, qui nous a rappelé les enjeux de cette plateforme d'excellence.

Mais Ogobara Doumbo, ce ne fut pas seulement cet « exemple » vivant d'un altruisme exceptionnel et concret, au service du continent africain. Il sut établir des liens multiples entre les hommes, entre les cultures et, notamment, avec les sciences, la médecine, la recherche pharmaceutique du pays, la France, qui nous réunit aujourd'hui ! Les marques et titres de reconnaissance que lui a value son œuvre en témoignent de façon éloquente.

Pour ma part, c'est au sein du conseil de la Fondation Mérieux que j'eus l'honneur de le rencontrer quelquefois, 
et d'apprécier chez lui ce mélange de détermination lucide et de modestie, ainsi que son esprit visionnaire.

C'est d'ailleurs à la grande générosité du Pr Alain Mérieux que les académies et instances scientifiques et médicales françaises doivent le soutien qui a permis la présente cérémonie. Je tiens à le remercier en notre nom à tous. Merci à nouveau à $\mathrm{M}$. le chancelier et à la Fondation Del Duca qui abrite cette réunion.

Plusieurs cérémonies à la mémoire du Pr Doumbo ont d'ailleurs précédé (à la Fondation Mérieux par exemple) l'hommage d'aujourd'hui. L'idée d'un témoignage de reconnaissance à la vie et à l'œuvre d'Ogobara Doumbo, dans un cadre proprement académique et universitaire, " germa » au sein du Coped que préside notre confrère Pierre Auger.
Bernard Surugue, qui connaissait bien le Pr Doumbo, fut en quelque sorte le "déclencheur ». Il m'est agréable de rappeler ici le rôle considérable qu'a joué notre éminent confrère académicien, Ghislain de Marsily, dans l'organisation et la conception de cette belle cérémonie d'hommage. Soulignons aussi des services de l'Académie, et notamment l'implication personnelle de Mme Anastasia Getskoff.

Bons périples à celles et ceux qui rejoignent leurs contrées d'origine. Notre reconnaissance va à toutes et à tous, et notamment aux membres de la famille Doumbo, particulièrement éprouvée, dont nous partageons la tristesse.

Que le souvenir du grand Africain, vrai « citoyen du monde ", que nous évoquons aujourd'hui, continue d'éclairer nos engagements !

Merci à tous! 